\title{
Targeted Nanobubbles Carrying Indocyanine Green for Ultrasound, Photoacoustic and Fluorescence Imaging of Prostate Cancer
}

This article was published in the following Dove Press journal: International Journal of Nanomedicine

\author{
Yixuan Wang (D) \\ Minmin Lan $^{2}$ \\ Daijia Shen ${ }^{2}$ \\ Kejing Fang ${ }^{2}$ \\ Lianhua Zhu (iD) ${ }^{2}$ \\ Yu Liu ${ }^{2}$ \\ Lan $\mathrm{HaO}^{3}$ \\ Pan Li (D) ${ }^{3}$
}

'The First Clinical College, Chongqing Medical University, Chongqing, People's Republic of China; ${ }^{2}$ Department of Ultrasound, Southwest Hospital, Army Medical University, Chongqing, People's Republic of China; ${ }^{3}$ Institute of Ultrasound Imaging, Chongqing Medical University, Chongqing, People's Republic of China
Correspondence: Pan Li Institute of Ultrasound Imaging, Chongqing Medical University, Chongqing, People's Republic of China

Email lipan@hospital.cqmu.edu.cn
Objective: To construct prostate-specific membrane antigen (PSMA)-targeting, indocyanine green (ICG)-loaded nanobubbles (NBs) for multimodal (ultrasound, photoacoustic and fluorescence) imaging of prostate cancer.

Methods: The mechanical oscillation method was used to prepare ICG-loaded photoacoustic NBs (ICG NBs). Then, PSMA-binding peptides were connected to the surface of ICG NBs using the biotin-avidin method to make targeted photoacoustic NBs, namely, PSMAP/ICG NBs. Their particle sizes, zeta potentials, and in vitro ultrasound, photoacoustic and fluorescence imaging were examined. Confocal laser scanning microscopy and flow cytometry were used to detect the binding ability of the PSMAP/ICG NBs to PSMA-positive LNCaP cells, C4-2 cells, and PSMAnegative PC-3 cells. The multimodal imaging effects of PSMAP/ICG NBs and ICG NBs were compared in nude mouse tumor xenografts.

Results: The particle size of the PSMAP/ICG NBs was approximately $457.7 \mathrm{~nm}$, and the zeta potential was approximately $-23.5 \mathrm{mV}$. Both confocal laser scanning microscopy and flow cytometry confirmed that the PSMAP/ICG NBs could specifically bind to both LNCaP and C4-2 cells, but they rarely bound to PC-3 cells. The ultrasound, photoacoustic and fluorescence imaging intensities of the PSMAP/ICG NBs in vitro positively correlated with their concentrations. The ultrasound and photoacoustic imaging effects of the PSMAP/ICG NBs in LNCaP and C4-2 tumor xenografts were significantly enhanced compared with those in PC-3 tumor xenografts, which were characterized by a significantly increased duration of ultrasound enhancement and heightened photoacoustic signal intensity $(\mathrm{P}<0.05)$. Fluorescence imaging showed that PSMAP/ICG NBs could accumulate in LNCaP and C4-2 tumor xenografts for a relatively long period.

Conclusion: The targeted photoacoustic nanobubbles prepared in this study can specifically bind to PSMA-positive prostate cancer cells and have the ability to enhance ultrasound, photoacoustic and fluorescence imaging of PSMA-positive tumor xenografts. Photoacoustic imaging could visually display the intensity of the red photoacoustic signal in the tumor region, providing a more intuitive imaging modality for targeted molecular imaging. This study presents a potential multimodal contrast agent for the accurate diagnosis and assessment of prostate cancer.

Keywords: prostate-specific membrane antigen, peptide, indocyanine green, targeted nanobubbles, ultrasound molecular imaging, photoacoustic imaging

\section{Introduction}

The early symptoms of prostate cancer are not obvious, and more than $60 \%$ of patients already have metastases when they are first diagnosed. Therefore, early detection of prostate cancer has important clinical significance for improving the survival rate and reducing the mortality of patients. ${ }^{1}$ Since digital rectal 
examination, serum prostate-specific antigen (PSA) detection, and imaging methods commonly used in clinical practice, including ultrasound, computed tomography (CT), and magnetic resonance imaging (MRI), cannot accurately differentiate and diagnose benign and malignant prostatic lesions, and since transrectal prostate biopsy is invasive and may lead to a missed diagnosis, developing a method that can accurately diagnose prostate cancer at an early stage has become an urgent task, representing a research hotspot in oncology and imaging studies. ${ }^{2-4}$

The advent of ultrasound molecular imaging has provided a new research field for the early diagnosis of tumors. Ultrasound molecular imaging mainly involves the use of microbubble contrast agents to visualize target tissues, and the ultrasound microbubbles currently used in clinical practice are usually micron-sized at $1-10 \mu \mathrm{m}$ in diameter. Since nascent blood vessels in tumors are structurally imperfect and the basement membrane is incomplete, an enhanced permeability and retention effect (EPR) occurs: that is, increased tumor vascular permeability permits certain macromolecules and particles to penetrate to the interstitial spaces and accumulate in tumor tissues. However, since the maximum pore size of the vessel wall is approximately $380-780 \mathrm{~nm}$, only substances with a particle size smaller than $700 \mathrm{~nm}$ can theoretically cross the walls of tumor blood vessels and enter the tumor interstitial space; therefore, conventional microbubbles cannot cross the blood vessel walls and enter the tumor tissue to truly achieve specific ultrasound imaging of the tumor parenchyma., Based on the tumor EPR, some scholars have constructed targeted nanobubbles (NBs) with diameters smaller than 700 $\mathrm{nm}$ and have studied their infiltration ability and specific ultrasound imaging effect. ${ }^{7,8}$ In previous studies, we successfully constructed a variety of targeted NBs against prostatespecific membrane antigen (PSMA) by linking monoclonal antibodies, nanobodies, and small aptamers to lipid NBs. The particle size of the NBs was approximately $500 \mathrm{~nm}$. In contrast to nontargeted NBs, the targeted NBs carrying PSMA ligands could specifically bind to prostate cancer cells in vitro and showed specific imaging characteristics such as a prolonged enhanced imaging time and increased peak intensity of tumor ultrasound in in vivo experiments, indicating that the targeted NBs loaded with PSMA ligands have a stronger penetration ability and higher specificity, which facilitates specific enhanced ultrasound imaging of parenchymal cells of prostate cancer and reflects a technical route and research basis for the targeted ultrasound molecular imaging of prostate cancer. ${ }^{9-11}$

The multimodal imaging technique involves the use of two or more imaging modalities to image the same object to obtain integrated information, thus facilitating the differentiation and diagnosis of tumors. ${ }^{12,13}$ Photoacoustic imaging (PAI) is an emerging biomedical imaging technology that has become a research hotspot in the field of biomedical imaging in recent years owing to its advantages of nonionization, noninvasiveness, high penetration, and low costs. Since different chemical components in tumor tissues have different optical absorption characteristics, PAI uses the light-induced ultrasound imaging mechanism to select specific laser wavelengths to detect chemical components in tumor tissues, allowing high-contrast structural imaging of tumor tissues. At the same time, hypermetabolism is an important hallmark of cancer that leads to changes in the hemoglobin concentration, blood oxygen concentration, and oxygen metabolic rate within tumor tissues. PAI allows the quantitative analysis of biological parameters such as the hemoglobin concentration, blood oxygen concentration, and oxygen metabolic rate within tumor tissues that can precisely reflect tiny tissue lesions, thereby enabling functional imaging of tumor tissues. ${ }^{14,15}$ Indocyanine green (ICG) has a strong ability to bind to plasma proteins in the vascular system and is the first optical contrast agent approved for clinical use by the US Food and Drug Administration (FDA) ${ }^{16,17}$ Another characteristic of ICG is its own red fluorescence, which can be used for fluorescence imaging.

We assume that the construction of targeted multifunctional NBs on the basis of ultrasound molecular imaging combined with the advantages of PAI may better facilitate ultrasound and photoacoustic molecular imaging and promote the early diagnosis of prostate cancer and efficacy evaluations. Since PSMA is highly expressed by malignant prostate epithelial cells, it represents an attractive candidate for selectively targeted ultrasound molecular imaging and targeted therapy for prostate cancer. Therefore, in this study, on the basis of our previous studies, we encapsulated ICG in the lipid shells of NBs and loaded PSMA ligands on the surfaces of NBs to construct targeted NBs for ultrasound, photoacoustic and fluorescence imaging of prostate cancer. Because PSMAbinding peptides have the advantages of PSMA-specific binding ability, low molecular weight, and low immunogenicity, peptides were selected as the binding ligand in this study.

\section{Materials and Methods Materials}

1,2-Dipalmitoyl-glycero-3 phosphate (DPPA), 1,2-dipalmitoyl-sn-glycero-3-phosphoglycerol (DPPG), 1,2-dipalmitoyl-sn-glycero-3-phosphatidic ethanolamine (DPPE) and 1,2-dipalmitoyl-sn-glycero-3-phosphocholine (DPPC) 
were purchased from Corden Pharma, Switzerland. Biotinylated 1,2-distearoyl-sn-glycero-3-phosphoethanolamine-PEG2000 (DSPE-PEG2000-Biotin) was purchased from NANOCS, USA. ICG was purchased from SigmaAldrich, USA. PSMA-binding peptides ${ }^{18,19}$ (sequence: WQPDTAHHWATLK(biotin)K(FITC)) were synthesized, purified and labeled by Sangon Biotech, Shanghai, China.

\section{Cell Culture and Xenograft Tumor Establishment in Nude Mice}

Human prostate cancer cell lines LNCaP (PSMA-positive) , C4-2 (PSMA-positive), and PC-3 (PSMA-negative) were all acquired from the ATCC cell bank (ATCC, USA) and cultured in DMEM (Thermo Fisher Scientific, USA) containing 10\% fetal bovine serum (Thermo Fisher Scientific), $100 \mathrm{U} / \mathrm{mL}$ penicillin, and $100 \mathrm{U} / \mathrm{mL}$ streptomycin in an incubator in the presence of $5 \% \mathrm{CO} 2$ at $37{ }^{\circ} \mathrm{C}$.

Four- to five-week-old male nude mice weighing 15-20 $\mathrm{g}$ were purchased from Beijing Huafukang Bioscience Co., Ltd., China. Two hundred microliters of log-phase LNCaP, C42 , and PC-3 cell suspensions were subcutaneously injected at a concentration of $5 \times 10^{6} / \mathrm{mL}$ into the backs of the nude mice to construct xenograft tumors. The animal experiments in this study were performed in accordance with NC3Rs ARRIVE guidelines and were approved by the Laboratory Animal Welfare and Ethics Committee of the Army Medical University, and the animals were cared for according to the Guidelines of the Army Medical University for Animal Welfare.

\section{Identification of PSMA Expression in Prostate Cancer Cells and Binding Ability of the PSMA-Binding Peptides to the Cells} Identification of PSMA expression LNCaP, C4-2, and PC-3 cells in logarithmic growth phase were seeded into laser confocal plates at a density of $5 \times 10^{4}$ cells $/ \mathrm{mL}$ and incubated overnight. The cells were fixed with $4 \%$ paraformaldehyde for $15 \mathrm{~min}$ and then blocked with $5 \%$ bovine serum albumin (BSA) for $2 \mathrm{~h}$. The cells were incubated with mouse antihuman PSMA monoclonal antibody (Abcam, UK) at $4{ }^{\circ} \mathrm{C}$ overnight and then with FITC-labeled goat anti-mouse IgG (Beyotime Biotechnology Company, China) in the dark at $37^{\circ}$ $\mathrm{C}$ for $2 \mathrm{~h}$. After the cells were washed with phosphate-buffered saline (PBS), they were counterstained with DAPI staining solution (Beyotime Biotechnology Company) for $5 \mathrm{~min}$. The cells were washed again, and the expression of PSMA on the surfaces of the three types of prostate cancer cells was observed under a confocal laser scanning microscope (CLSM, Zeiss, Germany).

Binding assay of PSMA-binding peptides Cells were prepared as above and incubated with FITC-labeled PSMA-binding peptides at $4{ }^{\circ} \mathrm{C}$ overnight. Then, the cells were washed with PBS and counterstained with DAPI staining solution for $5 \mathrm{~min}$. The cells were washed again and observed under a CLSM.

\section{Preparation and Characterization of the Targeted Photoacoustic Nanobubbles}

Targeted photoacoustic nanobubbles were prepared using the membrane hydration method. According to the ratio $3: 3: 3: 1: 1$, a total of $11 \mathrm{mg}$ of DPPC, DPPE, DPPG, DPPA, and DSPEPEG2000-Biotin as well as $1 \mathrm{mg}$ of ICG were dissolved in $1000 \mu \mathrm{L}$ of PBS/glycerol solution. After the agents were completely dissolved, the solution was transferred to a small vial. After displacement of the air in the vial with perfluoropropane $\left(\mathrm{C}_{3} \mathrm{~F}_{8}\right)$ (Tianjin Institute of Physics and Chemistry Engineering, China), the vial was shaken horizontally for 90 $\mathrm{s}$ in an ST amalgam capsule amalgamator (AT \& M Biomaterials Co., China). After standing for $2 \mathrm{~h}$, the entire solution in the vial was transferred to a $10-\mathrm{mL}$ centrifuge tube and centrifuged at $300 \mathrm{rpm} / \mathrm{min}$ for $3 \mathrm{~min}$. After centrifugation, the middle emulsion layer contained the prepared ICG-loaded NBs (ICG NBs). Streptavidin (Solarbio Science \& Technology Co. Ltd., Beijing, China) was added to the ICG NBs at a ratio of $3 \mu \mathrm{g} / 10^{7} \mathrm{ICG}$ NBs, followed by incubation for $1 \mathrm{~h}$ at $4{ }^{\circ} \mathrm{C}$. The NBs were washed three times, and then biotinylated PSMA-binding peptides were added to the NBs at a ratio of $0.32 \mu \mathrm{g} / 10^{7}$ ICG NBs, followed by incubation for $1 \mathrm{~h}$. The NBs were washed three times to obtain the PSMA-binding peptideand ICG-loaded targeted NBs (PSMAP/ICG NBs). The above processes were all protected from light.

The NBs were diluted 100-fold with PBS and added dropwise onto a hemocytometer, which was then placed under an inverted light microscope to observe and photograph the NBs. ImageJ software (imagej.nih.gov) was used to calculate the concentration of NBs. The distribution and morphology of the NBs were observed under a light microscope (Olympus, Japan) and a transmission electron microscope (JEOL, Japan). The particle sizes, size distributions, and zeta potentials of the NBs were measured by a Zetasizer Nano ZS90 particle size analyzer (Malvern Instruments Inc., UK). The NBs were observed and measured daily over a period of one week to evaluate changes in concentration and particle diameter with time (during this time, they were stored at $4^{\circ} \mathrm{C}$ ). 
ICG content in PSMAP/ICG NBs was measured in triplicate by a UV-Vis spectrophotometer (Thermo Fisher, USA). The absorbance of non-entrapped ICG in the supernatant was measured at $780 \mathrm{~nm}$. The mass of entrapped ICG was equal to mass of total ICG minus the mass of non-entrapped. The encapsulation efficiency (EE) and the drug loading efficiency (LE) of ICG were calculated. EE = the amount of ICG entrapped inside the NBs/the total amount of ICG added $\times$ $100 \%, \mathrm{LE}=$ the amount of ICG entrapped inside the NBs/the total amount of lipid used to prepare the NBs $\times 100 \%$.

The absorption spectra of free ICG, ICG NBs, and PSMAP/ICG NBs were obtained using a UV-Vis spectrophotometer.

As the ICG encapsulated in the NBs had its own red fluorescence and the peptides were labeled with FITC, the coupling of PSMA-binding peptides to NBs was verified directly under a CLSM.

Logarithmically growing PC-3, LNCaP, and C4-2 cells were seeded in 96-well culture plates and cultured for 24 $\mathrm{h}$ before the addition of different concentrations of PSMAP/ ICG NBs. The cells were then cultured for another $24 \mathrm{~h}$. The toxic effects of the PSMAP/ICG NBs on prostate cancer cells were assessed using Cell Counting Kit-8 (CCK-8) reagent (Beyotime Biotechnology Company).

\section{In vitro Cell Assay to Detect the Targeted Binding Ability of the PSMAP/ICG NBs}

Logarithmically growing LNCaP, C4-2, and PC-3 cells were seeded in 24-well plates at a density of $5 \times 10^{4}$ cells $/ \mathrm{mL}$ and cultured overnight. After culturing overnight, LNCap, C4-2, and PC-3 cells were each separated into three groups, fixed in $4 \%$ paraformaldehyde for 15 mins and subsequently blocked in $5 \%$ BSA-PBS at $37^{\circ} \mathrm{C}$ for $1 \mathrm{~h}$. One group was incubated with $30 \mu \mathrm{L}$ of PSMA/ICG NBs $\left(1 \times 10^{7} / \mathrm{mL}\right)$, one group was incubated with $30 \mu \mathrm{L}$ of ICG NBs $\left(1 \times 10^{7} / \mathrm{mL}\right)$, and another group was reacted with $100 \mu \mathrm{L}$ of mouse anti-PSMA monoclonal antibody $\left(1 \mu \mathrm{g} / \mathrm{mL}\right.$, Abcam) at $37{ }^{\circ} \mathrm{C}$ for $1 \mathrm{~h}$ before incubation with $30 \mu \mathrm{L}$ of PSMA/ICG NBs $\left(1 \times 10^{7} / \mathrm{mL}\right)$. After incubation at $37{ }^{\circ} \mathrm{C}$ for $1 \mathrm{~h}$, unbound nanobubbles were removed, the nuclei were counterstained with DAPI, and the binding ability of targeted and nontargeted nanobubbles to the tumor cells in vitro was observed under a CLSM.

Flow cytometry was used to further analyze the binding of PSMAP/ICG NBs and ICG NBs to prostate cancer cells. LNCaP, C4-2, and PC-3 cells in the logarithmic growth phase were dissociated with trypsin and collected into Eppendorf tubes at a concentration of $5 \times 10^{5}$ cells/tube, and the cells were resuspended in $500 \mu \mathrm{L}$ of PBS. Each of the three cell types was divided into three groups. The first group was used as a control and contained only cells. In the second group, the cells were mixed with $500 \mu \mathrm{L}$ of ICG NBs at a concentration of $2.0 \times 10^{7} \mathrm{NBs} / \mathrm{mL}$. In the third group, the cells were mixed with $500 \mu \mathrm{L}$ of PSMAP/ICG NBs at a concentration of $2.0 \times 10^{7} \mathrm{NBs} / \mathrm{mL}$. The mixtures were mixed gently. The PSMA-binding peptides of the PSMAP/ ICG NBs were labeled with FITC, and the mixtures were incubated at $37{ }^{\circ} \mathrm{C}$ for $40 \mathrm{~min}$. After centrifugation, the supernatant was removed. The cells were resuspended in $250 \mu \mathrm{L}$ of PBS, and the affinity of the NBs for the cells was measured by a FACSCalibur flow cytometer (BD Biosciences, USA).

\section{In vitro Imaging of PSMAP/ICG NBs}

PSMAP/ICG NBs and ICG NBs at concentrations of $1.0 \times 10^{8}$, $5.0 \times 10^{7}, 1.0 \times 10^{7}, 5.0 \times 10^{6}$, and $1.0 \times 10^{6} \mathrm{NBs} / \mathrm{mL}$ were placed in a $1 \%$ gel well model. Ultrasound images were acquired using a Vevo 2100 small-animal ultrasound imaging system (VisualSonics Inc., Canada), scanning with an MS250 high-frequency probe (bandwidth 13-24 MHz, central frequency $21 \mathrm{MHz}$ ). DFY ultrasonic image quantitative analyzer (Institute of Ultrasound Molecular Imaging, Chongqing, China) was used to carry out quantitative analysis on the images, and the gray value was calculated for statistical analysis.

The photoacoustic instrument used for this study was the Vevo LAZR (VisualSonics Inc., Canada), equipped with a transducer (Vevo LAZR LZ250) with a broadband ultrasound frequency of $13 \mathrm{MHz}-24 \mathrm{MHz}$, producing an axial resolution of $75 \mu \mathrm{m}$. For all the PA in vitro experiments, ultrasound frequency was set at $21 \mathrm{MHz}$, PA gain at $40 \mathrm{Db}$. Photoacoustic spectra were acquired in the wavelength range from $680 \mathrm{~nm}$ to $970 \mathrm{~nm}$ with a step size of $5 \mathrm{~nm}$. Two hundred PSMAP/ICG NBs at a concentration of $1.0 \times 10^{8} / \mathrm{mL}$ was placed in the $1 \%$ gel well model, and the best photoacoustic excitation wavelength was determined by a full wavelength scanning with the photoacoustic transducer. Then, photoacoustic images of PSMAP/ICG NBs at concentrations of $1.0 \times 10^{8}$, $5.0 \times 10^{7}, 1.0 \times 10^{7}, 5.0 \times 10^{6}$, and $1.0 \times 10^{6} \mathrm{NBs} / \mathrm{mL}$ were acquired using the best photoacoustic excitation wavelength. Photoacoustic image analysis was performed with the VevoLAB software package (VisualSonics). The signal intensity within the regions of interest (ROIs) was reported as photoacoustic signal.

The relationship between the ultrasound gray value and the photoacoustic signal value of the PSMAP/ICG NBs and their concentration was further analyzed. 
PSMAP/ICG NBs and ICG NBs were placed in an IVIS small-animal live imaging system (PerkinElmer, USA). Fluorescence images were scanned with a $740-\mathrm{nm}$ excitation wavelength and an $820-\mathrm{nm}$ emission wavelength, and the fluorescence intensities of the three groups were quantitatively analyzed using the Living Image IVIS software.

\section{In vivo Imaging of PSMAP/ICG NBs}

Five xenograft-bearing nude mice were randomly selected for ultrasound, photoacoustic, or fluorescence imaging when the volume of the three types of prostate cancer xenografts reached $100 \mathrm{~mm}^{3}$.

The nude mice were anesthetized and fixed, the maximum cross-section of the xenograft tumor was selected, and the imaging system was set to contrast-enhanced ultrasound mode (the mechanical index was 0.12 , and the frequency of the probe was $18 \mathrm{MHz}$ ). After randomized injection of $100 \mu \mathrm{L}$ of PSMAP/ICG NBs or ICG NBs at a concentration of $1 \times 10^{8}$ $\mathrm{NBs} / \mathrm{mL}$ via the posterior orbital venous sinus, contrastenhanced ultrasound images of the prostate cancer xenografts were acquired continuously and dynamically using a Vevo 2100 small-animal ultrasound imaging system. After the in vivo enhanced ultrasound imaging effect disappeared, another type of NB was injected in the same manner, and the time-intensity curves of the contrast-enhanced ultrasound images of the two types of NBs were quantitatively analyzed.

PAI of the prostate cancer xenografts in the nude mice was performed using a Vevo LAZR photoacoustic imaging system. After the PAI parameters (PA gain $44 \mathrm{~dB}$, the wavelength was $825 \mathrm{~nm}$, and the frequency at the center of the probe was 21 MHz) were adjusted, $100 \mu \mathrm{L}$ of PSMAP/ICG NBs or ICG NBs at a concentration of $1 \times 10^{8} \mathrm{NBs} / \mathrm{mL}$ was randomly injected via the posterior orbital venous sinus, and the photoacoustic signal of the xenograft tumor was collected continuously and dynamically. After the in vivo PAI signal disappeared, another type of NB was injected, photoacoustic images were collected in the same manner, and the time-photoacoustic signal intensity curves of the two NBs were quantitatively analyzed.

Fluorescence images of the nude mice before NB injection were collected using an IVIS small-animal live imaging system (the excitation and emission wavelengths were set at $740 \mathrm{~nm}$ and $820 \mathrm{~nm}$, respectively). Then, 100 $\mu \mathrm{L}$ of PSMAP/ICG NBs or ICG NBs at a concentration of $1 \times 10^{8} \mathrm{NBs} / \mathrm{mL}$ was injected into the nude mice via the posterior orbital venous sinus, and fluorescence images were collected at different time periods after injection.
Localization of PSMAP/ICG NBs in Tumor Tissues

Five nude mice bearing tumors of the three types of prostate cancer were randomly selected and $100 \mu \mathrm{L}$ of PSMAP/ICG NBs at a concentration of $1 \times 10^{8} \mathrm{NBs} / \mathrm{mL}$ was injected into the tumor-bearing nude mice via the posterior orbital venous sinus. The anesthetized nude mice were sacrificed 3 min later using cardiac perfusion with saline to remove all blood and concurrently all circulating nanobubbles. The xenograft tumor tissues were removed to create frozen sections. After the sections were fixed with paraformaldehyde, they were washed 3 times with PBS and blocked with 3\% BSA. Rabbit anti-mouse CD31 antibody (Abcam, UK) (1:300) was added, followed by incubation overnight at $4{ }^{\circ} \mathrm{C}$. After washing the sections with PBS, $100 \mu \mathrm{L}$ of FITC-labeled goat anti-rabbit secondary antibody (Beyotime Biotechnology Company) (1:300) was added, followed by incubation for $2 \mathrm{~h}$. DAPI staining solution was used for counterstaining for $5 \mathrm{~min}$. After washing, the sections were observed under a CLSM.

\section{Statistical Analysis}

One-way analysis of variance (ANOVA) and independentsamples t-tests were performed using Social Pack for Social Sciences 22.0. Measurement data are expressed as the mean \pm standard deviation. $\mathrm{P}<0.05$ was considered statistically significant. GraphPad Prism 6.0 was used to prepare line plots and histograms.

\section{Results \\ PSMA Expression in Prostate Cancer Cells and Binding Ability of PSMA-Binding Peptides to the Cells}

We used the immunocytofluorescence technique to detect the expression of PSMA protein on the cell membranes of LNCaP, C4-2, and PC-3 prostate cancer cells. Under a CLSM, we found that PSMA protein was highly expressed on the surfaces of LNCaP and C4-2 prostate cancer cells but was not expressed on the surfaces of PC-3 prostate cancer cells (Figure 1).

When the PSMA-binding peptides were labeled with FITC, observation by CLSM showed that the peptides were able to bind to $\mathrm{LNCaP}$ cells and $\mathrm{C} 4-2$ cells, while they did not bind to PC-3 cells (Figure 2), demonstrating that the PSMA-binding peptides synthesized in this study could specifically bind to PSMA-positive cells but not to PSMA-negative cells. 


\section{Characterization of the PSMAP/ICG NBs}

The schematic structure of PSMAP/ICG NBs was shown in Scheme 1. PSMAP/ICG NBs were perfluoropropane containing lipid nanoparticles. Its lipid shell contained ICG and biotinylated DSPE-PEG2000. The latter could combine with streptavidin, and the biotinylated PSMAbinding peptides are then attached to the streptavidin.

The PSMAP/ICG NBs were uniformly distributed and similar in size and shape and showed no aggregation under a light microscope (Figure 3A). Under transmission electron microscopy, the PSMAP/ICG NBs were spherical with smooth boundaries (Figure 3B). The particle size and zeta potential of the PSMAP/ICG NBs were $457.7 \pm$ $19.41 \mathrm{~nm}$ and $-23.5 \pm 0.4583 \mathrm{mV}$, respectively, as measured by the Zetasizer Nano ZS90 particle size analyzer (Figure 3C). The concentration of PSMAP/ICG NBs was $(13.6 \pm 0.23) \times 10^{8} \mathrm{NBs} / \mathrm{mL}$, and the concentration of ICG NBs was $(13.9 \pm 0.29) \times 10^{8} \mathrm{NBs} / \mathrm{mL}$. When the NBs were stored at $4{ }^{\circ} \mathrm{C}$ until the second day, there was no significant difference in concentration and particle size compared with day $0(\mathrm{P}>0.05)$. However, when the NBs were stored for $72 \mathrm{~h}$, there was a significant difference in concentration and particle size compared with day 0 $(\mathrm{P}<0.05)$. On the 7 th day of storage, the underlying liquid became visibly cloudy, indicating that some nanobubbles decomposed and broke down. The concentration decreased significantly, and the particle size increased significantly (Figure 3D). In addition, it was calculated that the EE and LE of ICG in PSMAP/ICG NBs were $70 \%$ and $(8.4 \pm$ $0.34) \%$, respectively.

The absorption spectra of free ICG, ICG NBs, and PSMAP/ICG NBs was shown in Figure 3E. The absorption spectra peaks of ICG NBs and PSMAP/ICG NBs were located at $780 \mathrm{~nm}$ as the free ICG solution. It showed that the preparation of NBs did not change the optical properties of ICG.

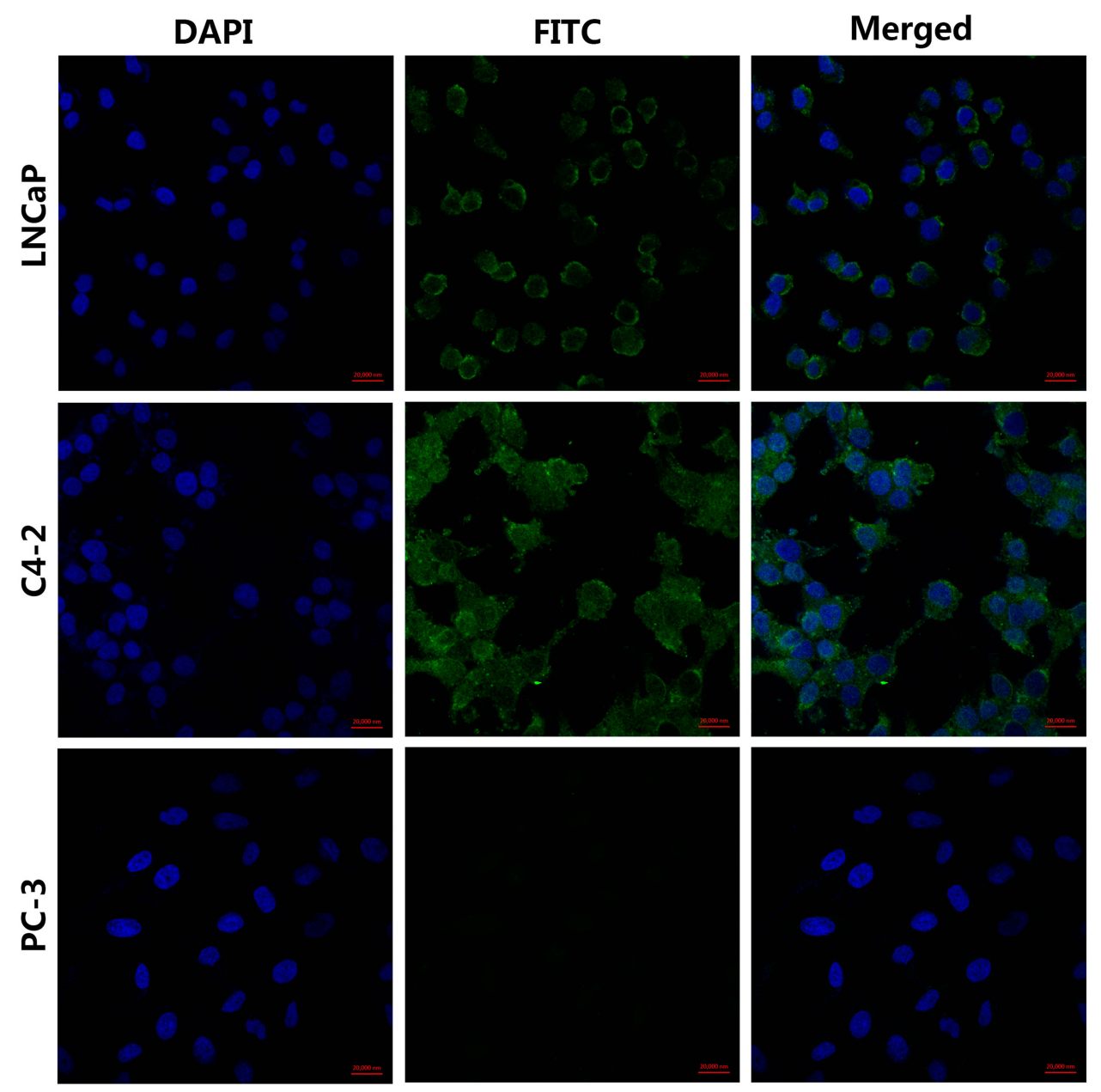

Figure I PSMA expression on the cell membranes of C4-2, LNCaP, and PC-3 prostate cancer cells Blue fluorescence represents nuclei, and green fluorescence represents PSMA. PSMA protein was highly expressed on the cell surfaces of LNCaP cells and C4-2 cells but was not expressed on the cell surfaces of PC-3 cells. 

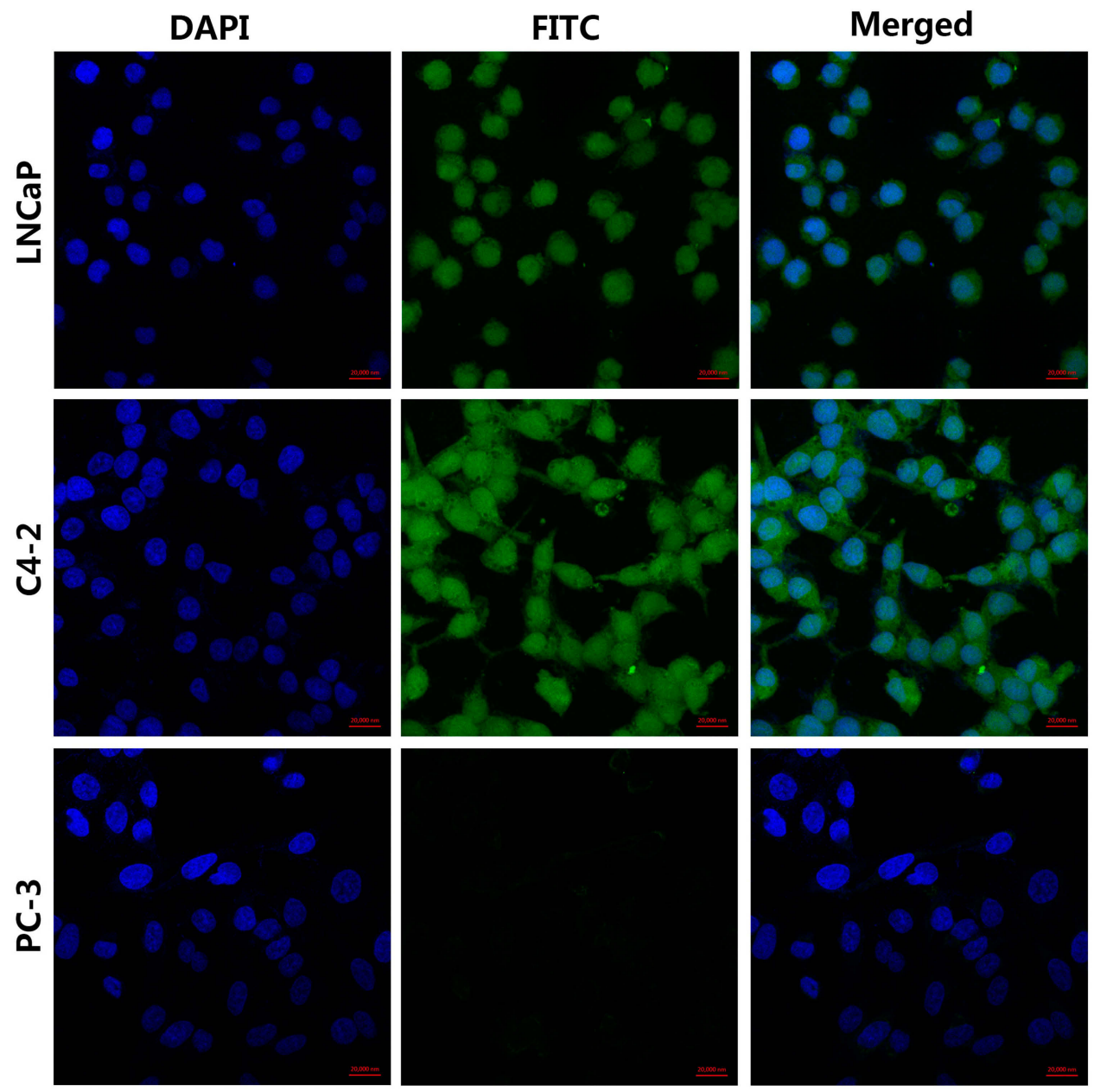

Figure 2 Binding ability of the PSMA-binding peptides to the cells Blue fluorescence represents the nuclei of the three types of prostate cancer cells, and green fluorescence represents the PSMA-binding peptides. The PSMA-binding peptides could specifically bind to PSMA-positive LNCaP cells and C4-2 cells but not to PSMA-negative PC-3 cells.

The toxic effects of the PSMAP/ICG NBs on LNCaP, C4-2, and PC-3 prostate cancer cells were analyzed using CCK-8. The results showed that in the concentration range of $1 \times 10^{6}$ to $1 \times 10^{8} \mathrm{NBs} / \mathrm{mL}$, PSMAP/ICG NBs at different concentrations did not significantly affect the cell viability of LNCaP, C4-2, and PC-3 prostate cancer cells $(\mathrm{P}>0.05)$. The effect of ICG NBs on cell viability was similar to that of the PSMAP/ICG NBs (Figure 3F).

Since the ICG encapsulated in the lipid shells of the PSMAP/ICG NBs had its own red fluorescence, the PSMAP/ICG NBs observed under a CLSM exhibited red fluorescence (Figure 4A). FITC-labeled PSMA-binding peptides showed green fluorescence (Figure 4B), and the complete overlap of the two types of fluorescence resulted in yellow fluorescence (Figure 4C), indicating that the PSMAbinding peptides were coupled to the surfaces of the PSMAP/ ICG NBs.

\section{Binding Capacity of PSMAP/ICG NBs to Prostate Cancer Cells}

After the LNCaP, C4-2, and PC-3 cells were incubated with the PSMAP/ICG NBs and ICG NBs, they were washed with PBS, and the binding of the PSMAP/ICG NBs and ICG NBs to the LNCaP, C4-2, and PC-3 prostate cancer cells was observed under a CLSM. A large number of PSMAP/ICG NBs accumulated at the periphery of the LNCaP and C4-2 cells (Figure 5A and D), while no obvious PSMAP/ICG NBs were present near the PC-3 cells (Figure 5G). After the LNCaP, C4-2, and PC-3 cells were incubated with the ICG NBs, only a small amount of the NBs accumulated around the cell membranes of all three types of prostate cancer cells (Figure 5B, E and H). When the cells were blocked with anti-PSMA monoclonal antibody, PSMAP/ICG NBs could not bind to any of the three types of cells (Figure 5C, F and I). These results 


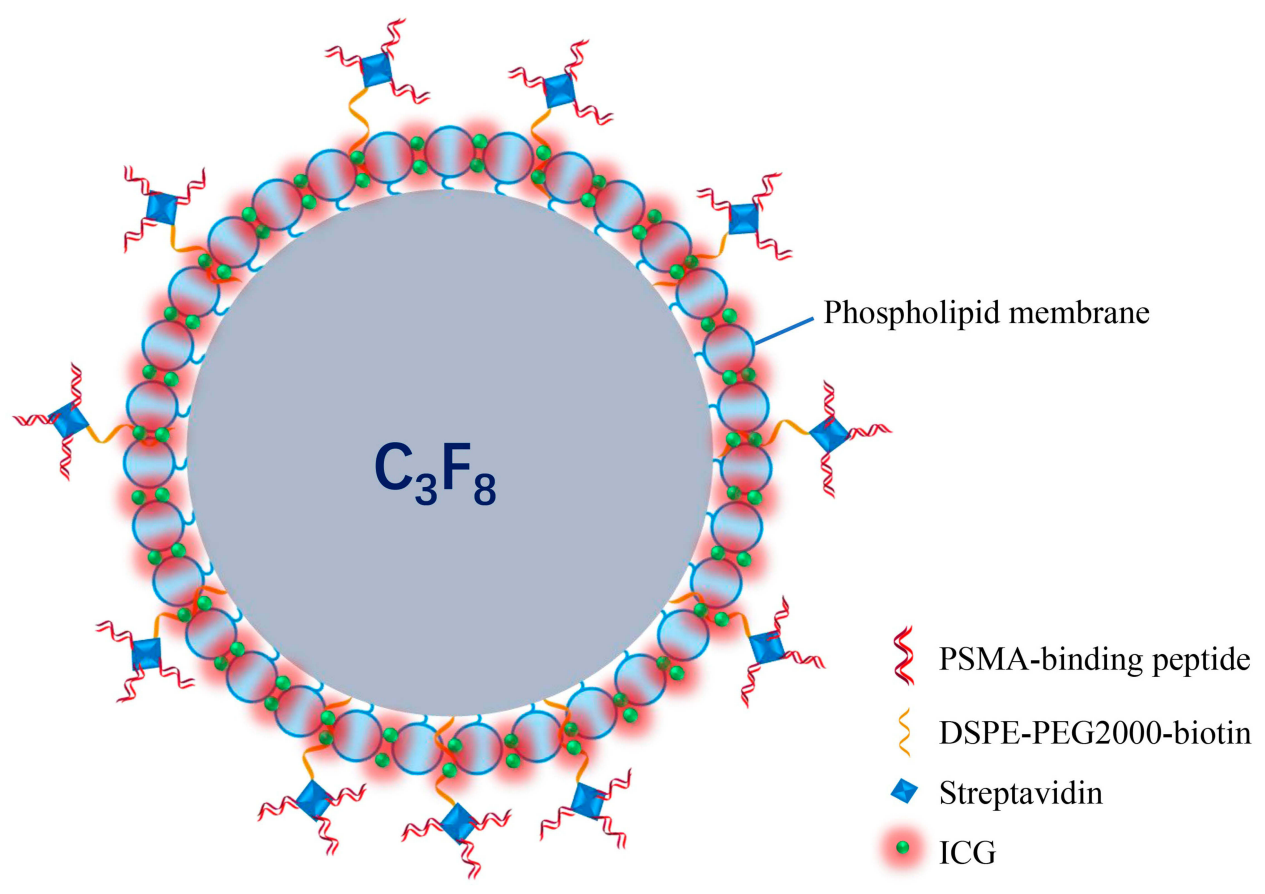

Scheme I The schematic illustration of PSMAP/ICG NBs.

Abbreviations: ICG, indocyanine green; $\mathrm{C}_{3} \mathrm{~F}_{8}$, perfluoropropane.

indicated that PSMAP/ICG NBs could specifically bind to PSMA-positive prostate cancer cells.

The binding ability of the PSMAP/ICG NBs and ICG NBs to the three types of prostate cancer cells was also assessed by flow cytometry. The affinity of the PSMAP/ICG NBs for LNCaP cells and C4-2 cells was significantly higher than that of the ICG NBs $(\mathrm{P}<0.01)$, whereas no significant difference in the affinity for PC-3 cells was found between the PSMAP/ICG NBs and ICG NBs $(P>0.05)$ (Figure 6).

\section{In vitro Imaging Capability of the PSMAP/ ICG NBs}

In vitro ultrasound imaging of PSMAP/ICG NBs at different concentrations were performed under contrast-enhanced ultrasound mode (the mechanical index was 0.12 , and the probe frequency was $18 \mathrm{MHz}$ ). Photoacoustic images of PSMAP/ ICG NBs at different concentrations were acquired using the best photoacoustic excitation wavelength $(825 \mathrm{~nm})$ and the value of photoacoustic signal intensity was analyzed quantitatively. In the in vitro agarose model, the ultrasound and PAI signal intensities of PSMAP/ICG NBs were positively correlated with their concentration, and both ultrasound and photoacoustic signal intensities were enhanced with increasing concentrations of PSMAP/ICG NBs (Figure 7A-C). An IVIS small-animal live imaging system was used to scan PSMAP/ ICG NB and ICG NB solutions with the same ICG concentrations. No significant difference in signal intensity was found between the PSMAP/ICG NBs and ICG NBs (P > 0.05 ) (Figure $7 \mathrm{D}$ and $\mathrm{E}$ ). The in vitro imaging results demonstrated that the PSMAP/ICG NBs possess ultrasound, photoacoustic and fluorescence multimodal imaging capabilities.

\section{In vivo Imaging of PSMAP/ICG NBs In vivo Ultrasound Imaging of PSMAP/ICG NBs}

Tumor tissues without injection of the NBs showed low echo. Injection of the PSMAP/ICG NBs and ICG NBs each resulted in good enhancement of ultrasound imaging, indicating that both types of NBs could penetrate tumor blood vessels and enhance tumor tissue imaging. Subsequently, continuous dynamic images were collected for $25 \mathrm{~min}$ (Figure 8A), the time-intensity curves (Figure 8B-D) were plotted, and the time to peak, peak intensity, duration time of enhancement, and area under the curve (AUC) were calculated (Figure 8E, Table 1). In PSMA-positive C4-2 and LNCaP xenograft tumors, the duration of enhancement and AUC differed significantly between the PSMAP/ICG NBs and ICG NBs $(\mathrm{P}<0.05)$, while the time to peak and peak intensity were not significantly different between the two types of NBs $(\mathrm{P}>0.05)$. At the same time, no significant differences in any of the above quantitative parameters were found between the PSMAP/ICG NBs and ICG NBs on enhanced ultrasound imaging of PSMAnegative PC-3 xenograft tumors $(\mathrm{P}>0.05)$. The above results 

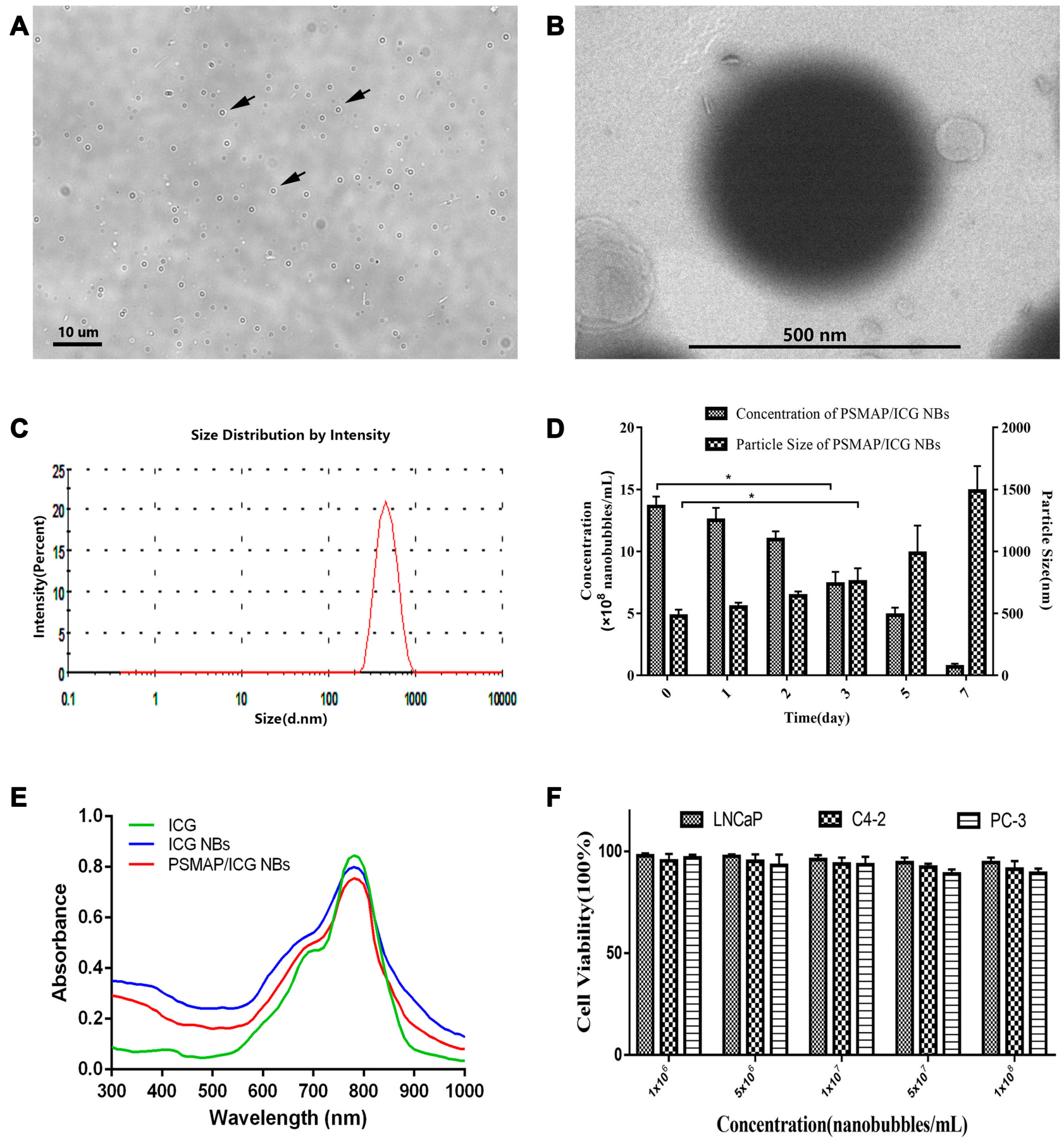

Figure 3 Basic characteristics of the PSMAP/ICG NBs (A) Morphology of the PSMAP/ICG NBs under light microscopy. (B) Morphology of the PSMAP/ICG NBs under transmission electron microscopy. (C) Particle size distribution of the PSMAP/ICG NBs; (D) Histogram showing the time dependence of changes in the concentration and particle diameter of the PSMAP/ICG NBs (* indicates P<0.05). (E) The absorption spectra of free ICG, ICG NBs, and PSMAP/ICG NBs. (F) The toxic effects of PSMAP/ICG NBs at different concentrations on the three types of prostate cancer cells.

indicated that compared with the ICG NBs, the PSMAP/ICG NBs significantly enhanced the ultrasound imaging of PSMApositive xenografts and that the difference was statistically significant. However, for PSMA-negative xenografts, the two types of NBs showed no significant difference.
Photoacoustic Imaging of the PSMAP/ICG NBs

After the PSMAP/ICG NBs and ICG NBs were injected into the C4-2, LNCaP, and PC-3 xenograft tumors, the photoacoustic signal intensities were significantly different from those before injection (Figure 9A). In 
ICG

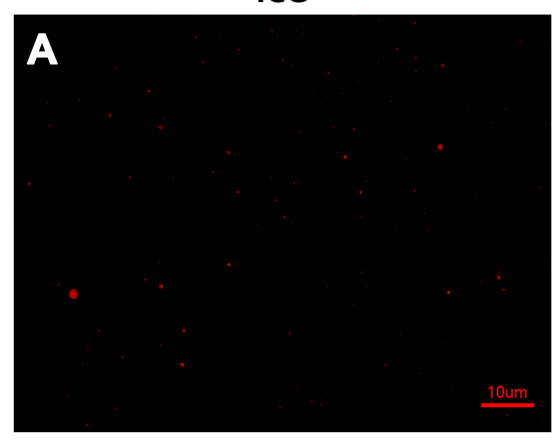

FITC

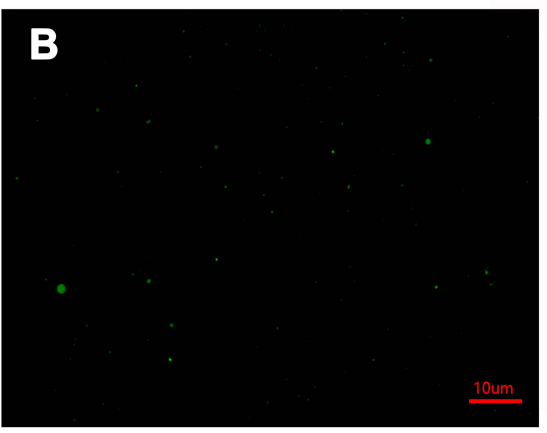

Merged

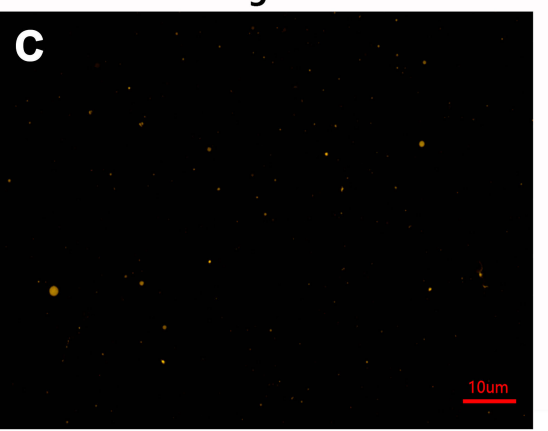

Figure 4 Verification of the coupling of PSMA-binding peptides to the surfaces of the PSMAP/ICG NBs When observed under a confocal laser scanning microscope, the NBs with encapsulated ICG in the lipid shell showed red fluorescence (A), and the FITC-labeled PSMA-binding peptides showed green fluorescence (B). The complete overlap of the two types of fluorescence resulted in yellow fluorescence (C).
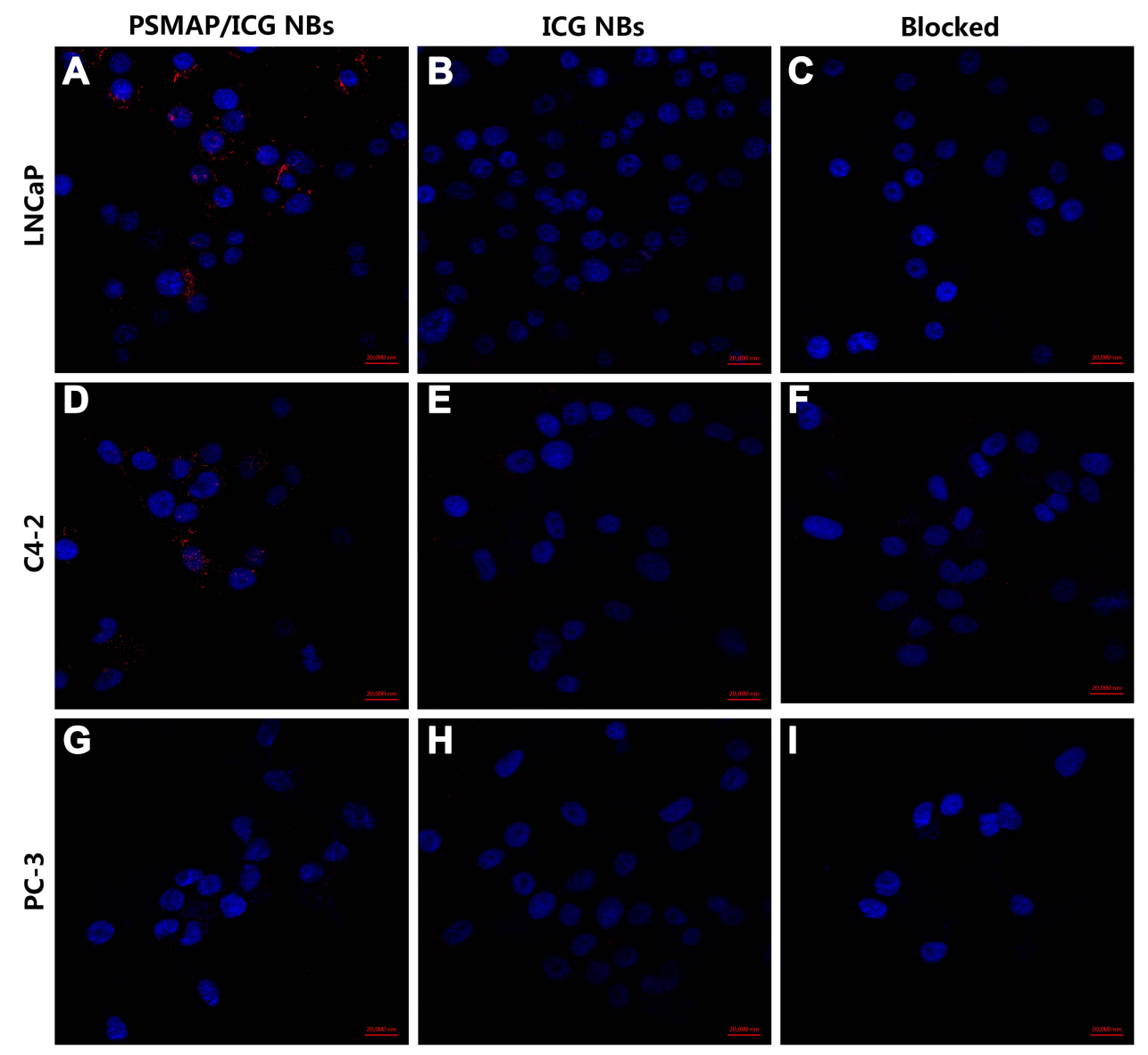

Figure 5 Binding capacity of the PSMAP/ICG NBs and ICG NBs to three types of prostate cancer cells Under CLSM, the nuclei of the three types of prostate cancer cells appeared blue, while the NBs appeared red. Aggregation of PSMAP/ICG NBs was observed around the membranes of LNCaP and C4-2 cells (A, D), while no obvious aggregation of ICG NBs was observed around the membranes of LNCaP and C4-2 cells (B, E). No significant accumulation of PSMAP/ICG NBs or ICG NBs was observed around the membranes of PC-3 cells $(\mathbf{G}, \mathbf{H})$. When the cells were blocked with anti-PSMA monoclonal antibody, PSMAP/ICG NBs could not bind to any of the three types of cells $(\mathbf{C}, \mathbf{F}, \mathbf{I})$.

particular, after injection of the PSMAP/ICG NBs, the photoacoustic signals in the $\mathrm{LNCaP}$ and $\mathrm{C} 4-2$ xenograft tumor areas were gradually enhanced, the PAI intensity was highest in the xenograft tumor areas at $5 \mathrm{~min}$ after injection, and the enhanced photoacoustic signal was still evident in the xenograft tumor areas 15 min after injection. By analyzing the photoacoustic signal intensities of the ICG NBs and PSMAP/ICG NBs in LNCaP, 

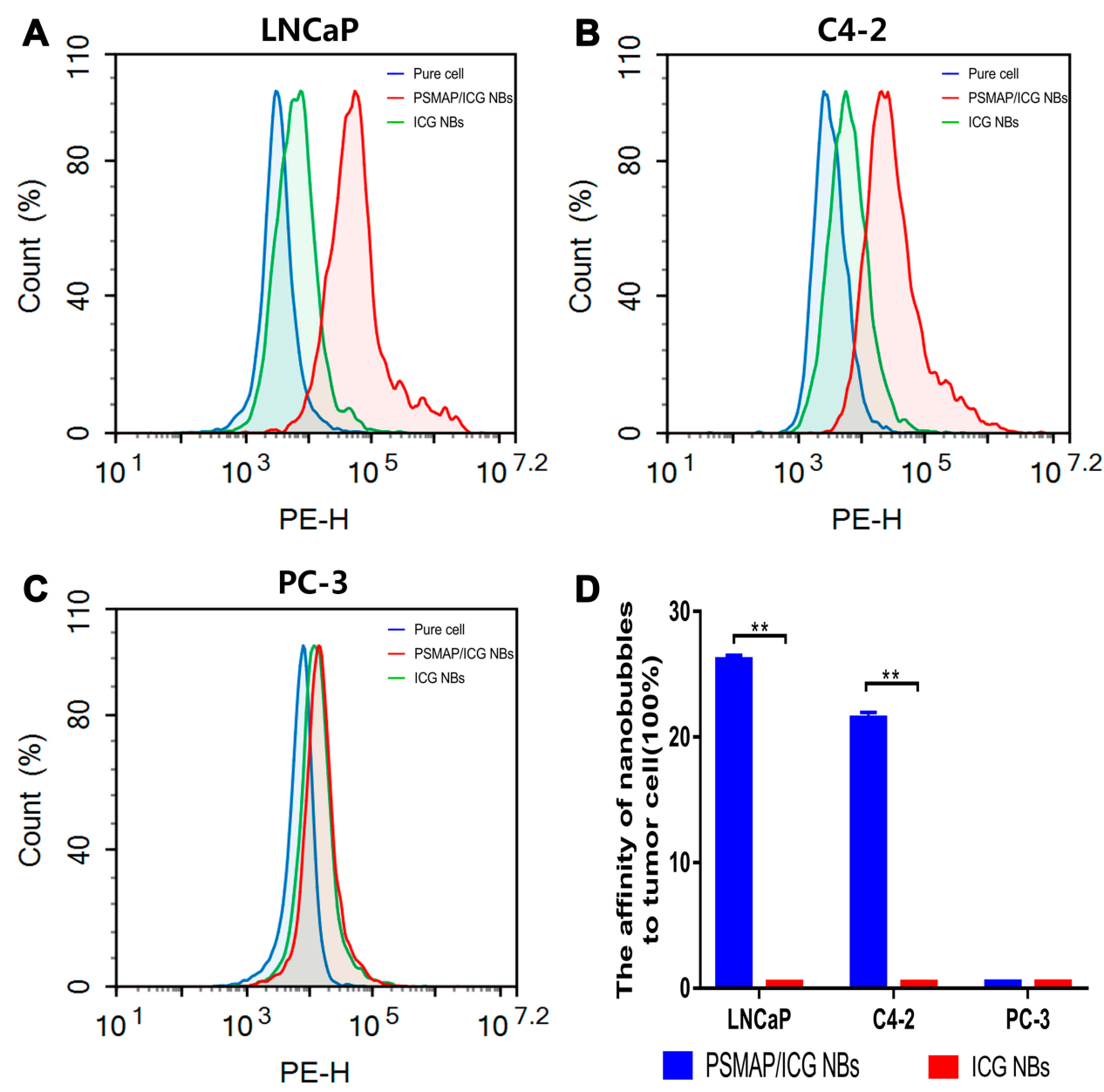

Figure 6 Detection of the affinity of PSMAP/ICG NBs and ICG NBs for cells by flow cytometry (A) LNCaP cells bound a large amount of PSMAP/ICG NBs but not ICG BNs. (B) C4-2 cells bound a large amount of PSMAP/ICG NBs but not ICG BNs. (C) PC-3 cells did not bind to any type of NB. (D) Quantification of the affinities of the two types of NBs for the three types of cells. ${ }^{* *}$ indicates $\mathrm{P}<0.01$.

C4-2, and PC-3 prostate cancer xenograft tumors, the photoacoustic signal intensities of the PSMAP/ICG NBs and ICG NBs were found to be significantly different in PSMA-positive C4-2 and LNCaP xenograft tumors $(\mathrm{P}<$ $0.05)$, while no significant difference in photoacoustic signal intensity was found between the PSMAP/ICG NBs and ICG NBs in PSMA-negative PC-3 xenograft tumors $(\mathrm{P}>0.05)$ (Figure $9 \mathrm{~B})$. In addition, the photoacoustic signal intensities of the PSMAP/ICG NBs in both C4-2 and LNCaP xenograft tumors were significantly higher than that of PSMAP/ICG NBs in PC-3 xenograft tumors $(\mathrm{P}<0.05)$, while the photoacoustic signal intensities of the ICG NBs were not significantly different in $\mathrm{C} 4-2, \mathrm{LNCaP}$, and $\mathrm{PC}-3$ prostate cancer xenograft tumors $(\mathrm{P}>0.05)$. These results indicated that targeted photoacoustic NBs could enhance the PAI intensity.

\section{Fluorescence Imaging of the PSMAP/ICG NBs}

Three minutes after injection of the PSMAP/ICG NBs or ICG NBs, obvious fluorescence signals were observed in the liver and the xenograft tumor. The fluorescence signal in the tumor began to gradually decay $15 \mathrm{~min}$ after injection, but a small amount of the fluorescence signal remained detectable after $60 \mathrm{~min}$. The time-fluorescence intensity curves and the AUCs showed a significant difference in fluorescence imaging results between PSMAP/ ICG NBs and ICG NBs in LNCaP and C4-2 xenograft tumors $(\mathrm{P}<0.05)$, indicating that the targeted PSMAP/ ICG NBs can accumulate in LNCaP and C4-2 xenograft tumors for a longer period and enhance the fluorescence imaging. No significant differences in the areas under the time-fluorescence intensity curves were found between the PSMAP/ICG NBs and ICG NBs in PC-3 xenograft tumors $(\mathrm{P}>0.05)$, further indicating that PSMAP/ICG 

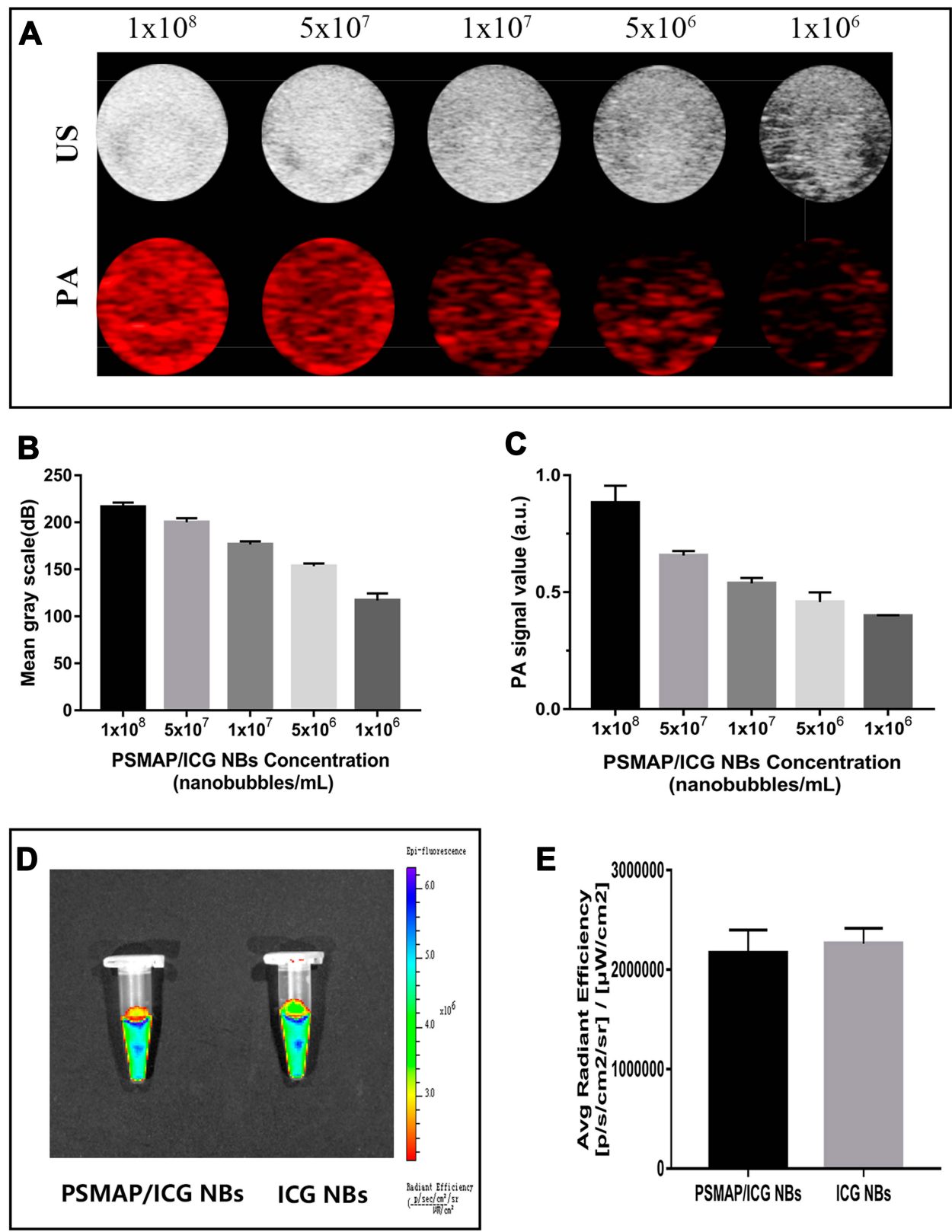

Figure 7 In vitro ultrasound, photoacoustic, and fluorescence imaging of PSMAP/ICG NBs (A) Ultrasound (US) and photoacoustic (PA) imaging of PSMAP/ICG NBs at different concentrations. (B) Quantification of US signal intensities at different PSMAP/ICG NB concentrations. (C) Quantification of PAI signal intensities at different PSMAP/ICG NB concentrations. (D) Fluorescence imaging of PSMAP/ICG NBs and ICG NBs in vitro. (E) Quantification of fluorescence signal intensity of the PSMAP/ICG $\mathrm{NBs}$ and ICG NBs in vitro.

NBs can accumulate in PSMA-positive prostate cancer tissues and specifically recognize prostate cancer cells (Figure 10, Table 2).

\section{Localization of PSMAP/ICG NBs in Tumor Tissues}

The penetration ability of PSMAP/ICG NBs was evaluated by immunohistofluorescence. In Figure 11, blue indicates cell nuclei, green indicates blood vessels in the tumor tissue, and red indicates ICG in the PSMAP/ICGNBs. It was showed that the red fluorescence of ICG in the targeted NBs was visible in the interstitial spaces of the three types of xenograft tumors. In the interstitial spaces of $\mathrm{LNCaP}$ and $\mathrm{C} 4-2$ prostate cancer xenograft tumors, a large number of PSMAP/ICG NBs accumulated around the $\mathrm{LNCaP}$ and $\mathrm{C} 4-2$ tumor cells (Figure 11A-H). Only a small number of PSMAP/ICG NBs were observed in the intercellular spaces of the PC-3 xenograft tumors (Figure 11I-L), and PSMAP/ICG NBs were barely 
A

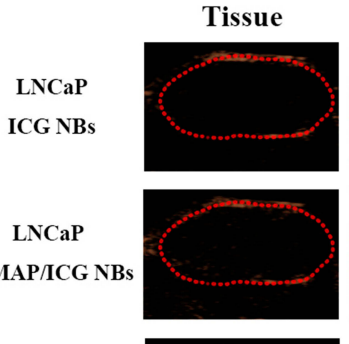

C4-2

ICG NBs

C4-2

PSMAP/ICG NBs

PC-3

ICG NBs

PC-3

PSMAP/ICG NBs
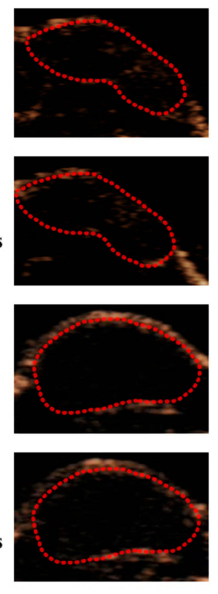

B

LNCaP

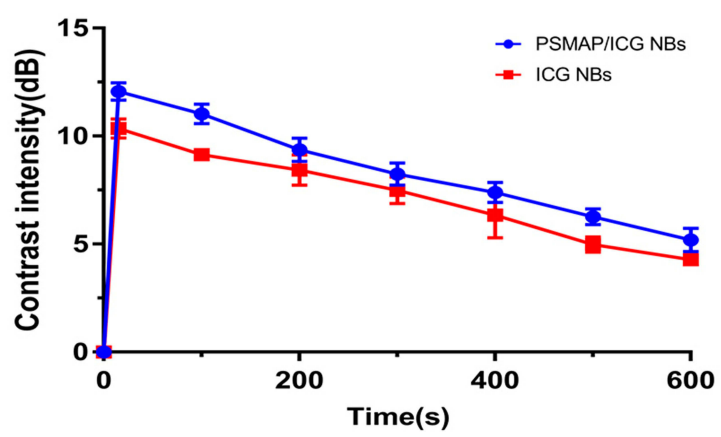

D
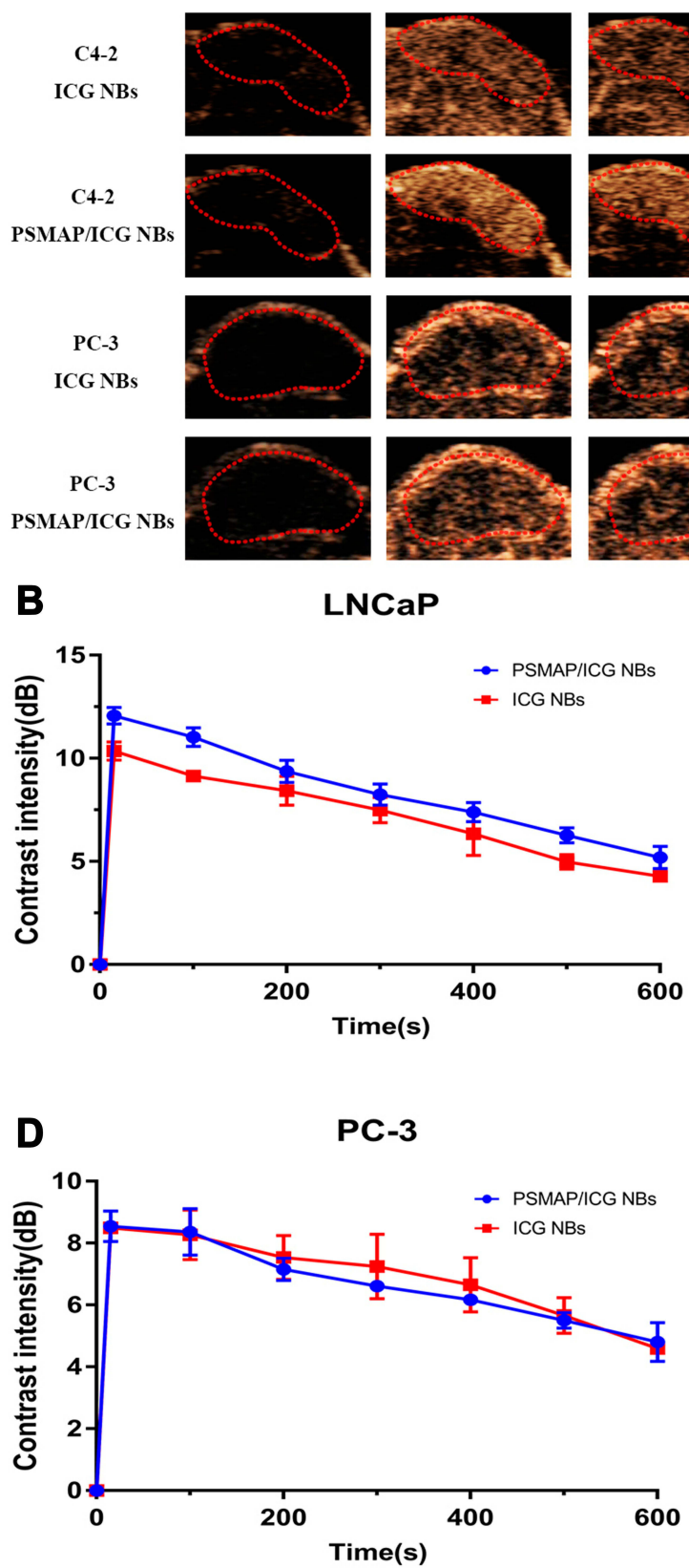
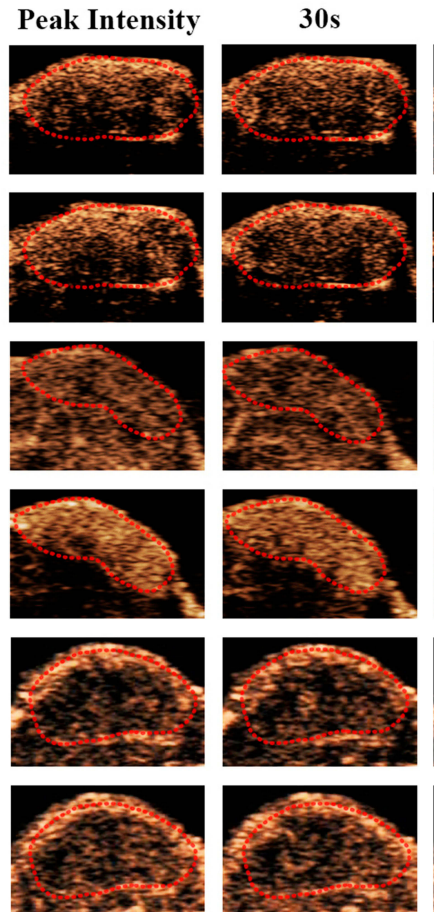

C
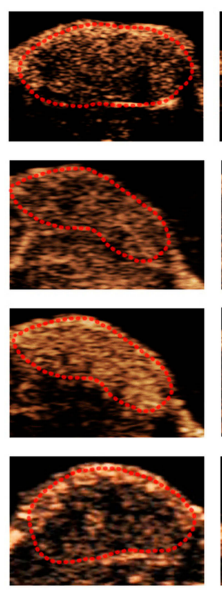
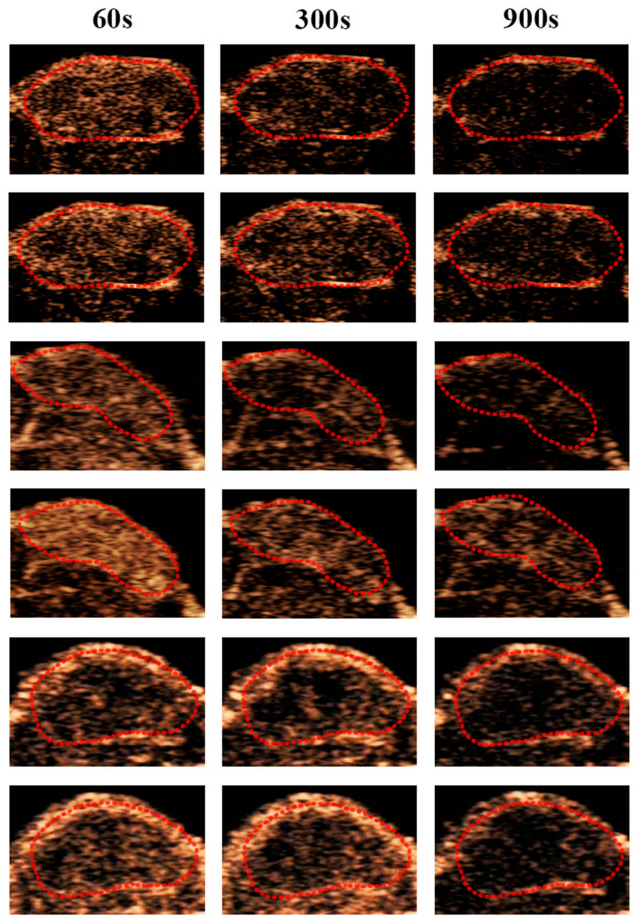

C4-2
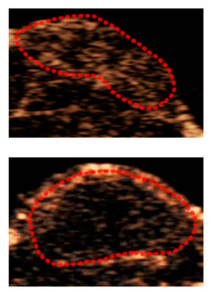

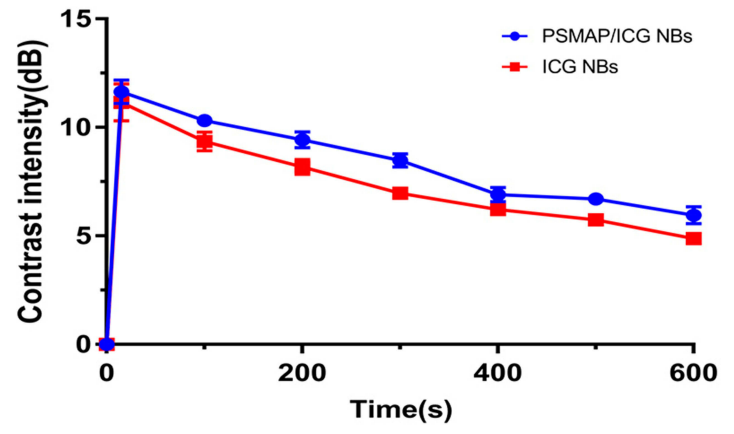

E

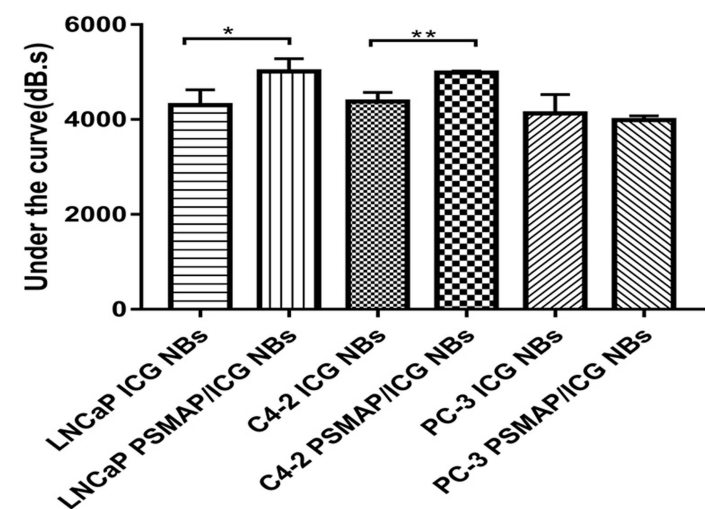

Figure 8 In vivo ultrasound imaging of PSMAP/ICG NBs and ICG NBs. The red dotted circles represent the area of prostate cancer xenograft tissues. (A) Ultrasound images of PSMA/ICG NBs and ICG NBs in the three types of xenograft tumors. (B-D): The time-intensity curves of ultrasound imaging of each xenograft tumor after injection of PSMA/ICG NBs and ICG NBs. (E) Calculation of the area under the time-intensity curve (AUC) and statistical analysis ( $n=5)$. *Indicates $P<0.05$, **indicates $\mathrm{P}<0.01$. 
Table I Ultrasound Parameters of PSMAP/ICG NBs and ICG NBs in Transplanted Tumors

\begin{tabular}{|l|l|l|l|l|l|}
\hline Tumor Type & Contrast Agents & Peak Time (s) & Peak Intensity (dB) & Duration Time (min) & AUC (dB.s) \\
\hline LNCaP & PSMAP/ICG NBs & $11.16 \pm 1.388$ & $11.68 \pm 0.5325$ & $21.83 \pm 1.089 *$ & $5012 \pm 157.1^{*}$ \\
& ICG NBs & $13.28 \pm 1.172$ & $10.18 \pm 0.2356$ & $15.57 \pm 0.4583$ & $4303 \pm 186.8$ \\
\hline C4-2 & PSMAP/ICG NBs & $12.48 \pm 1.977$ & $15.05 \pm 1.956$ & $22.51 \pm 0.489 *$ & $4986 \pm 20.58^{*}$ \\
& ICG NBs & $15.67 \pm 2.909$ & $12.86 \pm 2.595$ & $15.96 \pm 0.687$ & $4378 \pm 111.9$ \\
\hline PC-3 & PSMAP/ICG NBs & $15.4 \pm 1.430$ & $9.907 \pm 2.381$ & $15.82 \pm 0.4782$ & $3986 \pm 54.37$ \\
& ICG-ICG NBs & $13.66 \pm 1.906$ & $9.343 \pm 1.781$ & $15.45 \pm 0.1762$ & $4130 \pm 229.1$ \\
\hline
\end{tabular}

Notes: *The same ultrasound imaging parameters for the same type of xenograft tumors differed significantly between PSMAP/ICG NBs and ICG NBs (P < 0.05).

visible outside the blood vessels of normal muscle tissues (Figure 11M-P). These results demonstrated that the targeted nanobubbles could penetrate the tumor blood vessels into the tissue space and accumulate around the PSMA-positive cells.

\section{Discussion}

Prostate cancer is one of the most common malignant tumors and seriously threatens the health of elderly men. Early detection of lesions, precise determination of tumor numbers and boundaries, and accurate assessment of the treatment effect are critical for improving the survival rate and reducing the mortality of patients. Current conventional imaging techniques, such as ultrasound, CT, and MRI, cannot detect prostate cancer lesions with high sensitivity and high specificity at an early stage and provide precise intraoperative guidance. $^{20,21}$ Ultrasound molecular imaging achieves specific imaging of the targeted site by linking targeting ligands (antibodies, peptides, etc.) to the surface of the ultrasound contrast agent, allowing the contrast agent to selectively bind to the receptor at the target site and accumulate, which improves the ultrasound signal contrast between the target area and normal tissues. Compared with other imaging techniques, ultrasound molecular imaging has the advantages of clearly displaying the anatomical structure of a lesion and its relationship with adjacent structures and being noninvasive and easy to perform. Ultrasound molecular imaging has become a research focus in the field of molecular imaging and has generated hope regarding the specific imaging of prostate cancer. ${ }^{22}$ PSMA, a glycoprotein specifically expressed on the surfaces of prostate cancer cells, is specifically and highly expressed in both androgen-dependent and androgen-independent prostate cancers. The expression level of PSMA in prostate cancer is positively correlated with the Gleason grade of prostate lesions. PSMA has become a common target in the molecular imaging of prostate cancer. ${ }^{23,24}$ Micron-scale ultrasound contrast agent is a blood pool contrast agent, has a relatively large particle size, and can dynamically display tumor microcirculation and perfusion in real time. However, the contrast agent cannot pass through tumor blood vessels and enter the tumor tissues, which substantially limits its scope of application. In our previous series of studies, we successively constructed multiple targeted NBs carrying PSMA monoclonal antibodies, nanobodies, and aptamers that can cross tumor blood vessels in prostate cancer. Studies have demonstrated that unlike conventional micron-scale ultrasound contrast agents, targeted NBs have an average particle size of approximately $500 \mathrm{~nm}$ and can penetrate tumor blood vessels and enter the prostate cancer tissue space. Compared with PSMA-negative prostate cancer, targeted NBs carrying PSMA ligands can cause PSMApositive prostate cancer lesions to exhibit specific features on enhanced ultrasound imaging, such as prolonged enhancement, increased peak intensity, and a larger area under the timeintensity curve. However, our study also found that because targeted enhanced ultrasound imaging is a form of grayscale imaging, it has several disadvantages: namely, the imaging effect is singular, the difference in specific ultrasound imaging of prostate cancer between targeted NBs and nontargeted NBs is not intuitive, quantitative analysis software is needed to determine the difference in ultrasound imaging between targeted NBs and nontargeted NBs, and the image analysis time is long, which is not conducive to rapid and accurate diagnosis and precise intraoperative guidance. ${ }^{9,11,25}$

Since various imaging techniques have their own imaging characteristics, no single-mode imaging technique has been found to be ideal. Multimodal imaging technology combines the advantages of multiple imaging techniques by integrating ultrasound, fluorescence, CT, magnetic resonance, and radionuclide techniques. ${ }^{26-28}$ For example, positron-emission tomography (PET)/CT and PET/MR imaging systems that have been applied in clinical practice can simultaneously provide more rapid high-resolution anatomical, functional, metabolic, or molecular information, thus facilitating broader 


\section{A Tumor (Pre)}
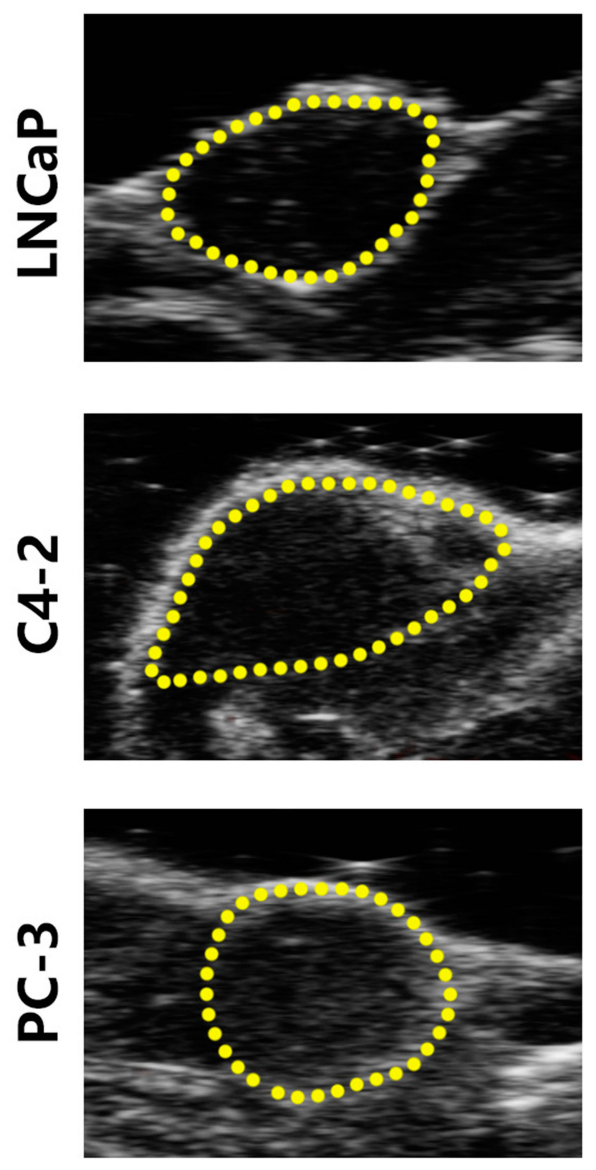

B

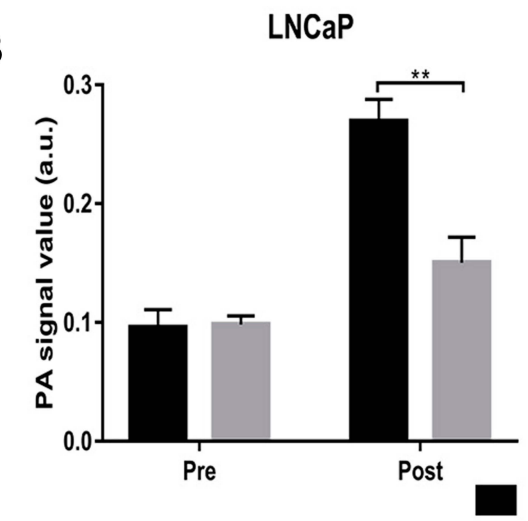

PSMAP/ICG NBs
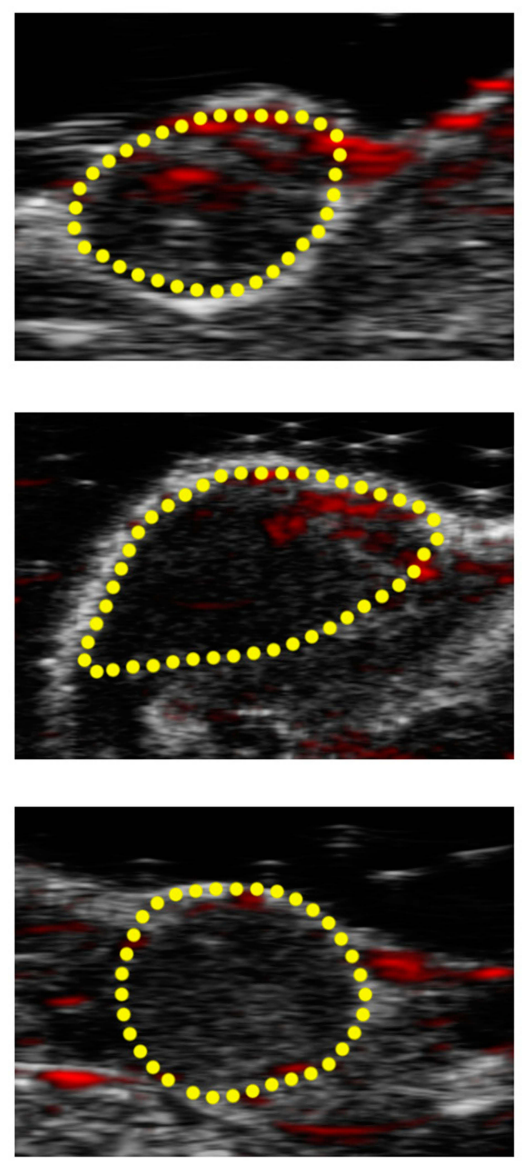

C4-2
ICG NBs
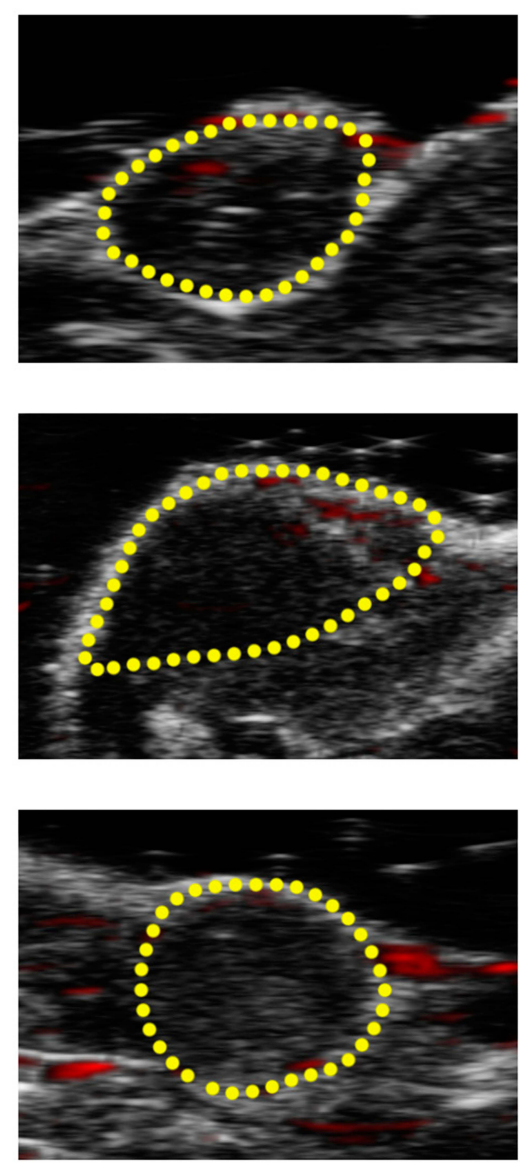

PC-3
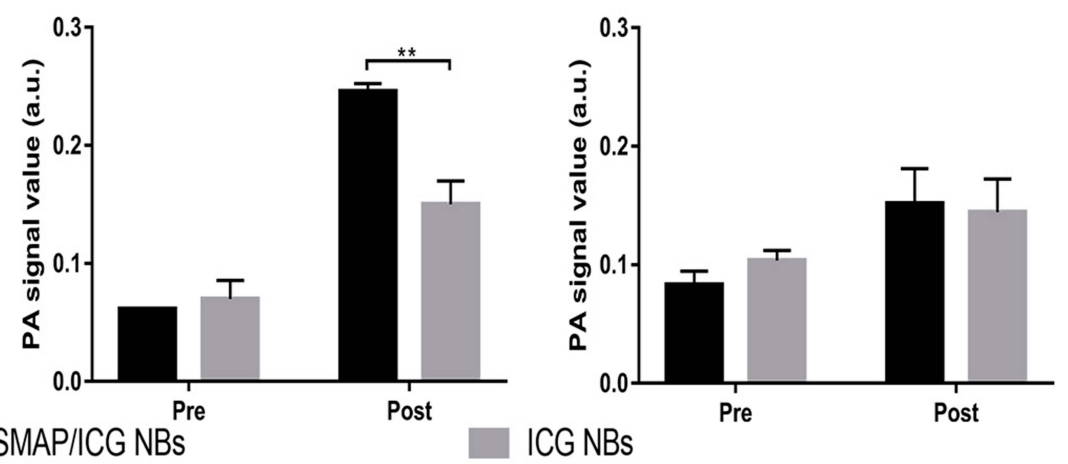

Figure 9 Photoacoustic imaging of the PSMAP/ICG NBs and ICG NBs. (A) Images of PAI of PSMAP/ICG NBs and ICG NBs pre- and post-injection. Inside the yellow dotted circles are the area of the transplanted tumors. The grey signal represents the routine ultrasound signal, and the red signal represents the PA signal. (B) Intensities of PAI of PSMAP/ICG NBs and ICG NBs pre- and post-injection $(n=5)$. **Indicates $P<0.01$.

research and application prospects for molecular imaging. PAI is a highly sensitive imaging technique developed in recent years that combines the high penetration depth of ultrasound imaging with high-contrast optical properties; therefore, PAI can uncover high-contrast and highresolution imaging effects for clinical use. PAI also has the advantages of no radioactivity and good safety performance.
In particular, PAI can quantitatively analyze biological parameters such as the hemoglobin concentration, blood oxygen concentration, and oxygen metabolic rate in tumor tissues, thereby accurately reflecting tissue microlesions and thus enabling the functional imaging of tumor tissues. PAI has great potential for application in tumor diagnosis and posttreatment evaluations. ICG is one of the few photoacoustic 


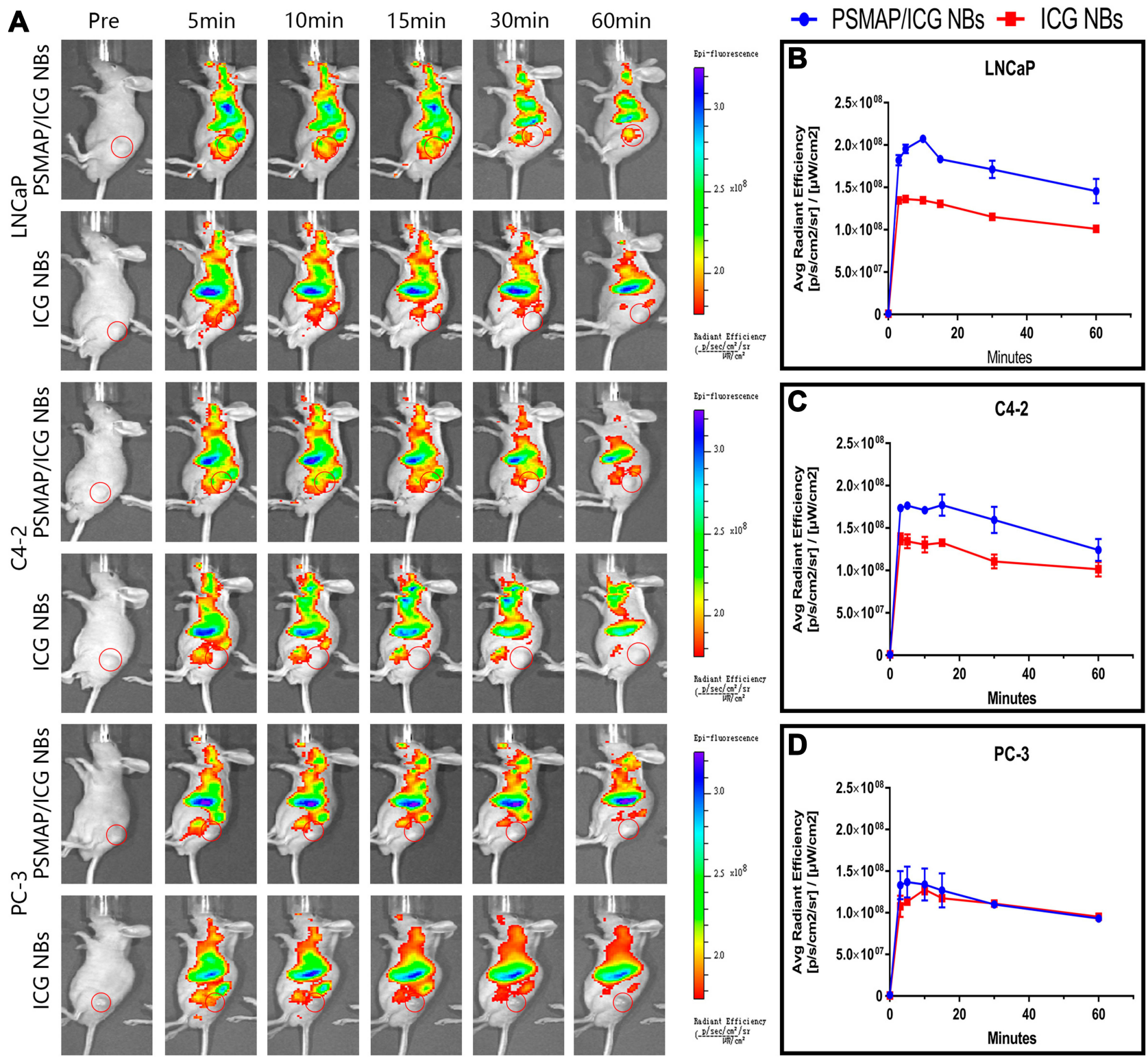

Figure 10 Small-animal live fluorescence imaging of the three types of prostate cancer xenograft tumors in nude mice Near-infrared fluorescence images of nude mice carrying LNCaP, C4-2, and PC-3 xenograft tumors after the injection of PSMAP/ICG NBs or ICG NBs (Inside the red circles are areas of xenograft tumors) (A); Timefluorescence intensity curves in tumor regions at different time points (B-D).

and fluorescent contrast agents approved for intraoperative clinical use by the US FDA, and available clinical and experimental results have shown that ICG-based PAI can

Table 2 Area Under the Time-Fluorescence Intensity Curves of PSMAP/ICG NBs and ICG NBs in Transplanted Tumors

\begin{tabular}{|l|l|l|l|}
\hline NBs & LNCaP & C4-2 & PC-3 \\
\hline PSMAP/ICG NBs (a.u.) & $100.55 \pm 2.54 *$ & $91.40 \pm 1.63^{*}$ & $66.31 \pm 2.78$ \\
ICG NBs (a.u.) & $69.11 \pm 0.55$ & $68.12 \pm 0.73$ & $64.13 \pm 0.54$ \\
\hline
\end{tabular}

Notes: *Represents a significant difference between the PSMAP/ICG NBs group and the ICG NBs group $(\mathrm{P}<0.05)$. play an important role in intraoperative tumor number and boundary determination and postoperative assessments. ${ }^{29,30}$ Because ICG is nontargeting and has a high affinity for plasma protein, it has good application prospects as a blood flow monitoring agent and can provide good oncologic imaging results. ${ }^{31}$ If ICG exists in the form of free molecules, problems such as poor water stability and rapid clearance from the body may arise, thus reducing its imaging time. Therefore, the construction of PAI-based multimodal molecular probes by linking ICG to other imaging contrast agents can prolong ICG imaging time and facilitate the accurate diagnosis of tumors. Relevant studies have shown that the 

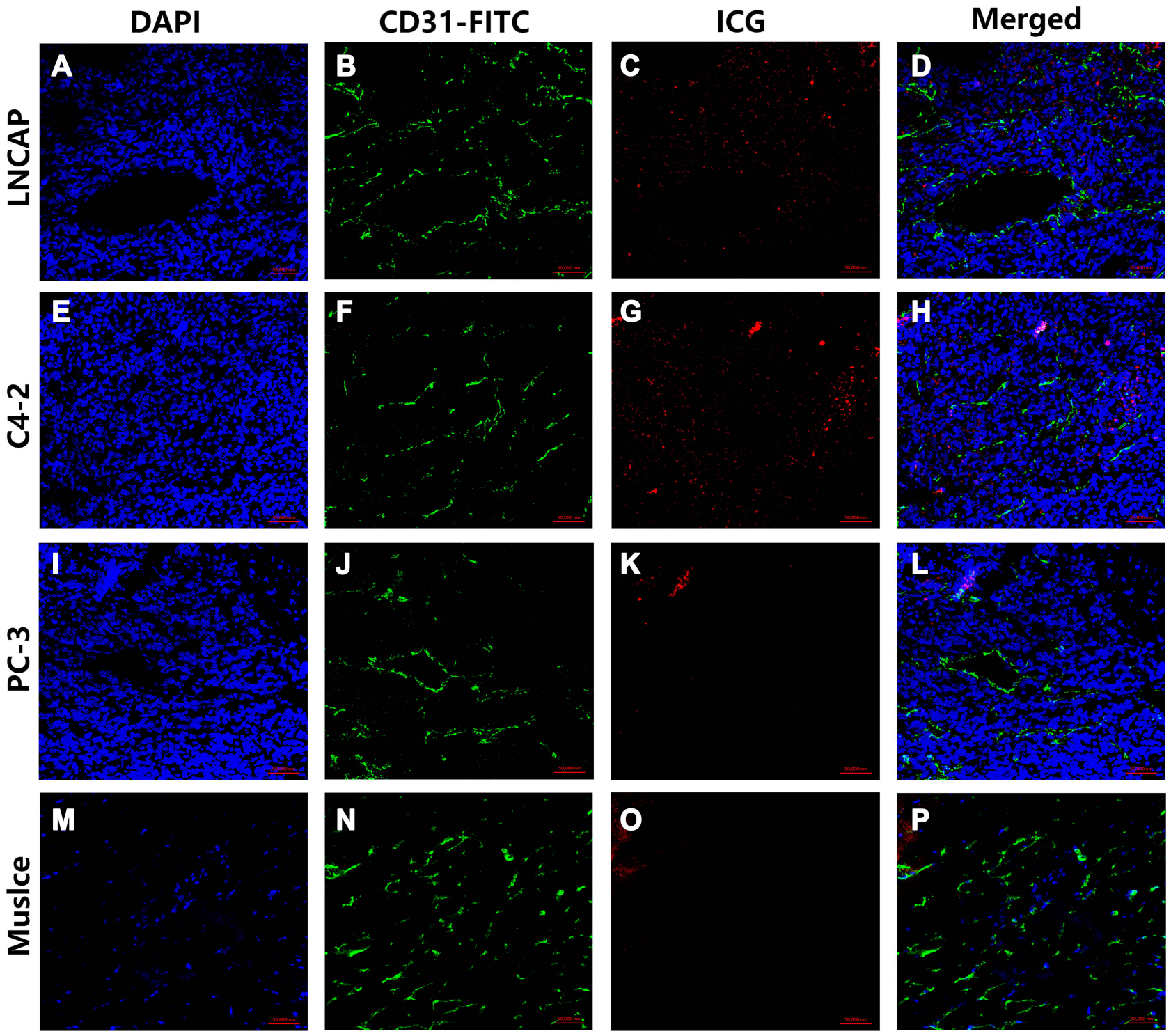

Figure II Localization of the PSMAP/ICG NBs in xenograft tumor tissues by immunohistofluorescence. The nuclei of the three types of prostate cancer appeared blue (DAPI), the blood vessels of the tumor appeared green (CD3I-FITC), and the PSMAP/ICG NBs appeared red (ICG). A large number of PSMAP/ICG NBs were observed in the intercellular spaces of $L N C a P$ and $C 4-2$ xenografts $(\mathbf{A}-\mathbf{H})$. A small number of PSMAP/ICG NBs were observed in the intercellular spaces of PC-3 xenografts (I-L), and PSMAP/ICG NBs were barely visible outside the blood vessels of normal muscle tissues (M-P).

encapsulation of ICG into liposomes can effectively improve the stability of ICG, prolong its circulation time in the blood, and increase its fluorescence and photoacoustic signals. ${ }^{28,32}$ Hannah A et al encapsulated ICG in the albumin shell of gascontaining nanodroplets, and achieved ultrasound and photoacoustic dual modal imaging. ${ }^{33}$ Considering the advantages and disadvantages of targeted NBs carrying PSMA ligands in prostate cancer imaging in our previous study and the advantages of PAI, we encapsulated ICG in the lipid shells of targeted NBs carrying PSMA-binding peptides to construct targeted NBs for multimodal (ultrasound, photoacoustic and fluorescence) imaging of prostate cancer in this study.
High-affinity anti-PSMA monoclonal antibodies include the J415, J533, and J591 series, which bind to the extracellular domain of PSMA. ${ }^{34}$ However, monoclonal antibodies have disadvantages such as high molecular weight, weak penetration in tumor tissues, and strong immunogenicity. Related studies have confirmed that compared with monoclonal antibodies, anti-PSMA short peptides, which specifically recognize molecules on the tumor cell surface, have numerous advantages, such as low molecular weight, easy expression and synthesis, low immunogenicity, high affinity and high specificity for tumors, and easy uptake by tumor cells; therefore, anti-PSMA short peptides are ideal prostate 
cancer-targeting molecules and have attracted increasing attention with respect to medical diagnosis and treatment. Therefore, a PSMA-binding peptide (sequence: WQPDTAHHWATL) was selected for this study. ${ }^{18,19}$ In vitro experiments confirmed that the peptides could specifically bind to the cell membranes of PSMA-positive LNCap and C4-2 cells. In in vitro targeted binding experiments, both CLSM and flow cytometry confirmed that PSMAP/ICG NBs could bind specifically to PSMA-positive LNCaP and C4-2 prostate cancer cells and that could not bind to PSMAnegative PC-3 prostate cancer cells.

The NBs prepared in this study was detected to ensure their characteristics was suitable for use. PSMAP/ICG NBs had spherical shapes, smooth boundaries, similar sizes and shapes, and even distributions, with a particle size of approximately $457.7 \mathrm{~nm}$ and a zeta potential of approximately $-23.5 \mathrm{mV}$. These characteristics were similar to those of the nanoparticles we have previously studied and prepared ${ }^{35,36}$ 。 The cytotoxicity assay showed that various concentrations of PSMAP/ICG NBs had no significant effect on the viability of PC-3, LNCaP, and C4-2 prostate cancer cells, indicating that they are sufficiently safe. Confocal laser scanning microscopy showed that the green fluorescence of the PSMA peptide on PSMAP/ICG NBs could sufficiently overlap with the red fluorescence of ICG to show yellow fluorescence, confirming that the PSMA peptide was successfully linked to the surfaces of the PSMAP/ ICG NBs. Therefore, the NBs prepared in this study met the design requirements and were suitable for ultrasound, photoacoustic and fluorescence imaging in vitro and in vivo.

Investigating the in vitro imaging capabilities of the nanoparticles is the basis of in vivo imaging studies. ${ }^{37,38}$ The in vitro imaging results in this study showed that PSMAP/ICG NBs could be used for simultaneous ultrasound, photoacoustic, and fluorescence multimodality imaging. The ultrasound imaging intensity and photoacoustic signal intensity of PSMAP/ICG NBs increased with their increasing concentration. This laid the foundation for in vivo experiments.

Early detection of a tumor and its metastases and clear delineation of the tumor number and margins during surgery are current difficulties and research hotspots in the imaging diagnosis of prostate cancer. With the development of highly specific PSMA ligands, preoperative and intraoperative multimodal imaging studies of prostate cancer have received increasing attention. In nuclear medicine, ${ }^{99 \mathrm{~m}} \mathrm{Tc}$ - and ${ }^{111} \mathrm{In}$ labeled small molecule PSMA ligands have been used in several clinical studies and are considered the most promising radiosurgery tracers for clinical applications. ${ }^{39-41}$ Lutje et al used the radioactive diagnostic drug ${ }^{111}$ In and the near-infrared fluorescent dye IR-800CW to label the anti-PSMA monoclonal antibody D2B, constructed a PET/CT and fluorescence multimodal tracer targeting PSMA, and investigated the tracer's potential in the PET/CT- and fluorescence imagingguided resection of PSMA-positive tumors. The results showed that both microPET/CT and fluorescence imaging modalities can clearly display PSMA-expressing LS174T tumors in the peritoneal cavity two days after intravenous injection of the multimodal tracer. However, among the LS174T-PSMA tumors at different depths in the peritoneal cavities of the mice, only the two most superficial tumors were evident due to the limited penetration depth of nearinfrared fluorescence imaging, while microSPECT/CT imaging showed several additional tumor lesions. The combination of the two imaging techniques has played an important role in intraoperative guidance, the accurate visualization of tumor number and morphology, and complete resection of tumors. ${ }^{42}$ However, compared with the multimodal imaging techniques in which SPECT/CT is combined with fluorescence imaging, the ultrasound imaging-based multimodal imaging method has the advantages of greater convenience, rapid execution, and intuitive imaging, facilitating its application in the operating room and at the bedside. ${ }^{43,44}$ Targeted ultrasound NBs with particle sizes between 400 and $700 \mathrm{~nm}$ have been found to cross tumor blood vessels and enter the interstitial spaces of tumors to achieve specific imaging of tumor parenchymal cells. $^{45,46}$ On this basis, we constructed PSMAP/ ICG NBs in this study by combining the advantages of PAI. Compared with nontargeted ICG NBs, the targeted PSMAP/ ICG NBs showed stronger ultrasound, photoacoustic and fluorescence imaging effects in PSMA-positive LNCaP and C4-2 xenograft tumor tissues. Quantitative analysis of the two types of xenograft tumors by ultrasound targeted imaging revealed that the PSMAP/ICG NBs had a longer enhanced ultrasound duration and a larger AUC. The reason may be that the particle sizes of the PSMAP/ICG NBs and ICG NBs were not different, and both NBs therefore achieved better penetration in $\mathrm{LNCaP}$ and C4-2 prostate cancer xenograft tumors. Accordingly, no significant differences in the peak time and peak intensity were found between PSMAP/ICG NBs and ICG NBs. However, unlike ICG NBs, PSMAP/ICG NBs had the ability to specifically bind to the cell membranes of the above two types of PSMA-positive prostate cancer xenograft tumors, which allowed them to specifically accumulate in the xenograft tissues for a long time, thereby effectively increasing the enhanced imaging duration and the area under the timeintensity curves in the above two types of xenograft tumors. 
PAI showed that the photoacoustic signal intensity of the PSMAP/ICG NBs was significantly higher than that of ICG NBs in LNCaP and C4-2 xenograft tumors. Moreover, the photoacoustic signal intensity of the PSMAP/ICG NBs in LNCaP and C4-2 xenograft tumors was significantly higher than that in PC-3 xenograft tumors. After injection of the PSMAP/ICG NBs, the photoacoustic signals in the $\mathrm{LNCaP}$ and $\mathrm{C} 4-2$ xenograft tumor regions gradually increased. The PAI effect was highest $5 \mathrm{~min}$ after injection, and the enhanced photoacoustic signal in the tumor region persisted 15 min after injection. Unlike targeted ultrasound imaging of tumors, which requires quantitative analysis of relevant parameters, PAI can more directly display the intensity and area of the red photoacoustic signal in the tumor region, providing a more intuitive imaging method for the targeted molecular imaging of prostate cancer. The results of the small-animal fluorescence imaging experiments further showed that the PSMAP/ICG NBs could accumulate in $\mathrm{LNCaP}$ and $\mathrm{C} 4-2$ prostate cancer xenograft tumors for a longer time than ICG NBs, but no significant differences in fluorescence intensity and duration were found between the two NBs in PC-3 xenograft tumors. Based on the results of the two imaging studies, the PSMAP/ICG NBs were further confirmed to specifically accumulate in the lesion area of PSMA-positive prostate cancer and specifically recognize PSMA-positive prostate cancer cells.

Compared with ultrasound, PAI provides intuitive and clear tissue contrast images with high sensitivity, which is conducive to accurate diagnosis and localization of tumors. ${ }^{29,30}$ Fluorescence imaging has the advantages of high sensitivity and intuitive image, but there are some shortcomings such as low spatial resolution and poor tissue permeability, and the localization of the lesion is inferior to that of ultrasound and photoacoustic. The multimodal imaging formed by the combination of photoacoustic, ultrasound and fluorescence imaging can directly and comprehensively obtain the tumor tissue structure and the molecular information of cell, which is conducive to the accurate early diagnosis, localization of tumors, and the visual evaluation of tumor areas after treatment.

Immunohistofluorescence assays of tumor tissues further confirmed that PSMAP/ICG NBs were distributed in the interstitial spaces of the LNCaP, C4-2, and PC-3 xenograft tumors, although greater accumulation was observed in the interstitial spaces of PSMA-positive LNCaP and C4-2 xenograft tumors, indicating that the PSMAP/ICG NBs had a better affinity for PSMA-positive LNCaP and C4-2 xenograft tumor tissues than for PSMA-negative PC-3 xenograft tumor tissues, which enabled them to accumulate in PSMA-positive xenograft tumor tissues. As a result, the number of NBs in local tumor tissues continuously increased, which specifically enhanced the ultrasound, photoacoustic and fluorescence imaging intensity of the tumor tissues and prolonged the enhanced imaging time, thus laying a pathomorphological foundation for PSMAP/ICG NBs to achieve multimodal molecular imaging of tumor tissues beyond the tumor blood vessels.

\section{Conclusion}

In this study, we constructed multimodal targeted NBs (PSMAP/ICG NBs) for prostate cancer by encapsulating the photoacoustic contrast agent ICG in the lipid shells and coupling PSMA-binding peptides to the surfaces of the NBs. The targeted photoacoustic NBs can specifically bind to PSMApositive prostate cancer cells and have the ability to enhance ultrasound, photoacoustic and fluorescence imaging of PSMApositive tumor xenografts. In particular, PAI could visually display the intensity of the red photoacoustic signal in the tumor region, providing a more intuitive imaging modality for targeted molecular imaging. This study not only presents a new targeted ultrasound, photoacoustic and fluorescence contrast agent for the accurate diagnosis of prostate cancer but also provides a research method and basis for the multimodal imaging of other tumors.

\section{Acknowledgments}

This work was supported by the National Natural Science Foundation of China (No. 81771856, No. 81771847) and the National International Science and Technology Cooperation Project (No. 2015DFA30920).

\section{Disclosure}

The authors declare that they have no competing interests, and the authors listed are solely responsible for the content of this study.

\section{References}

1. ReferenceSiegel RL, Miller KD, Jemal A. Cancer statistics, 2016. $C A$ Cancer J Clin. 2016;66(1):7-30. doi:10.3322/caac.21332

2. Mottet N, Bellmunt J, Bolla M, et al. EAU-ESTRO-SIOG guidelines on prostate cancer. Part 1: screening, diagnosis, and local treatment with curative intent. Eur Urol. 2017;71(4):618-629. doi:10.1016/j. eururo.2016.08.003

3. Yossepowitch O, Briganti A, Eastham JA, et al. Positive surgical margins after radical prostatectomy: a systematic review and contemporary update. Eur Urol. 2014;65(2):303-313. doi:10.1016/j. eururo.2013.07.039 
4. Porres D, Pfister D, Thissen A, et al. The role of salvage extended lymph node dissection in patients with rising PSA and PET/CT scan detected nodal recurrence of prostate cancer. Prostate Cancer Prostatic Dis. 2017;20(1):85-92. doi:10.1038/pcan.2016.54

5. Unger E, Porter T, Lindner J, et al. Cardiovascular drug delivery with ultrasound and microbubbles. Adv Drug Deliv Rev. 2014;72:110-126. doi:10.1016/j.addr.2014.01.012

6. Boissenot T, Bordat A, Fattal E, et al. Ultrasound-triggered drug delivery for cancer treatment using drug delivery systems: from theoretical considerations to practical applications. $J$ Control Release. 2016;241:144-163. doi:10.1016/j.jconrel.2016.09.026

7. Wu M, Wang Y, Wang Y, et al. Paclitaxel-loaded and A10-3.2 aptamer-targeted poly(lactide-co-glycolic acid) nanobubbles for ultrasound imaging and therapy of prostate cancer. Int J Nanomedicine. 2017;12:5313-5330. doi:10.2147/IJN.S136032

8. Prabhakar A, Banerjee R. Nanobubble liposome complexes for diagnostic imaging and ultrasound-triggered drug delivery in cancers: a theranostic approach. ACS Omega. 2019;4(13):15567-15580. doi:10.1021/acsomega.9b01924

9. Wang L, Li L, Guo Y, et al. Construction and in vitro/in vivo targeting of PSMA-targeted nanoscale microbubbles in prostate cancer. Prostate. 2013;73(11):1147-1158. doi:10.1002/pros.22663

10. Fan X, Guo Y, Wang L, et al. Diagnosis of prostate cancer using anti-PSMA aptamer a10-3.2-oriented lipid nanobubbles. Int J Nanomedicine. 2016;11:3939-3950. doi:10.2147/IJN.S112951

11. Fan X, Wang L, Guo Y, et al. Ultrasonic nanobubbles carrying anti-PSMA nanobody: construction and application in prostate cancer-targeted imaging. PLoS One. 2015;10(6):e0127419. doi:10.1371/journal.pone.0127419

12. Lutje S, Rijpkema M, Helfrich W, et al. Targeted radionuclide and fluorescence dual-modality imaging of cancer: preclinical advances and clinical translation. Mol Imaging Biol. 2014;16(6):747-755. doi:10.1007/s11307-014-0747-y

13. Sasikumar A, Kashyap R, Joy A, et al. Utility of 68Ga-PSMA-11 PET/CT in imaging of glioma - a pilot study. Clin Nucl Med. 2018;43 (9):e304-e309. doi:10.1097/RLU.0000000000002175

14. Liu Y, Lu X, Liu H, et al. Porous gold nanocluster decorated manganese monoxide nanocomposites for microenvironment-activatable $\mathrm{MR} /$ photoacoustic/CT tumor imaging. Nanoscale. 2018;10 (8):3631-3638. doi:10.1039/C7NR08535D

15. Neuman BP, Eifler JB, Castanares M, et al. Real-time, near-infrared fluorescence imaging with an optimized dye/light source/camera combination for surgical guidance of prostate cancer. Clin Cancer Res. 2015;21(4):771-780. doi:10.1158/1078-0432.CCR-14-0891

16. Jones AD, Wilton JC. Can intra-operative fluorescence play a significant role in hepatobiliary surgery? Eur J Surg Oncol. 2017;43(9):1622-1627. doi:10.1016/j.ejso.2017.02.015

17. Vahrmeijer AL, Hutteman M, van der Vorst JR, et al. Image-guided cancer surgery using near-infrared fluorescence. Nat Rev Clin Oncol. 2013;10(9):507-518. doi:10.1038/nrclinonc.2013.123

18. Aggarwal S, Singh P, Topaloglu O, et al. A dimeric peptide that binds selectively to prostate-specific membrane antigen and inhibits its enzymatic activity. Cancer Res. 2006;66(18):9171-9177. doi:10.1158/0008-5472.CAN-06-1520

19. Zhang H, Liu X, Wu F, et al. A novel prostate-specific membrane-antigen (PSMA) targeted micelle-encapsulating wogonin inhibits prostate cancer cell proliferation via inducing intrinsic apoptotic pathway. Int J Mol Sci. 2016;7(5):E676. doi:10.3390/ijms17050676

20. Heidenreich A, Bellmunt J, Bolla M, et al. EAU guidelines on prostate cancer. Part 1: screening, diagnosis, and treatment of clinically localised disease. Eur Urol. 2011;59(1):61-71. doi:10.1016/j. eururo.2010.10.039

21. Claeys T, Van Praet C, Lumen N, et al. Salvage pelvic lymph node dissection in recurrent prostate cancer: surgical and early oncological outcome. Biomed Res Int. 2015;2015:198543. doi:10.1155/2015/ 198543
22. Zhu L, Wang L, Liu Y, et al. CAIX aptamer-functionalized targeted nanobubbles for ultrasound molecular imaging of various tumors. Int J Nanomedicine. 2018;13:6481-6495. doi:10.2147/IJN.S176287

23. Mannweiler S, Amersdorfer P, Trajanoski S, et al. Heterogeneity of prostate-specific membrane antigen (PSMA) expression in prostate carcinoma with distant metastasis. Pathol Oncol Res. 2009;15 (2):167-172. doi:10.1007/s12253-008-9104-2

24. Afshar-Oromieh A, Babich JW, Kratochwil C, et al. The rise of PSMA ligands for diagnosis and therapy of prostate cancer. $J \mathrm{Nucl}$ Med. 2016;57(Supplement_3):79s-89s. doi:10.2967/ jnumed.115.170720

25. Fan X, Wang L, Guo Y, et al. Inhibition of prostate cancer growth using doxorubicin assisted by ultrasound-targeted nanobubble destruction. Int J Nanomedicine. 2016;11:3585-3596. doi:10.2147/ IJN.S111808

26. Banerjee SR, Pullambhatla M, Byun Y, et al. Sequential SPECT and optical imaging of experimental models of prostate cancer with a dual modality inhibitor of the prostate-specific membrane antigen. Angew Chem Int Ed Engl. 2011;50(39):9167-9170. doi:10.1002/ anie. 201102872

27. Hekman MC, Rijpkema M, Muselaers $\mathrm{CH}$, et al. Tumor-targeted dual-modality imaging to improve intraoperative visualization of clear cell renal cell carcinoma: a first in man study. Theranostics. 2018;8(8):2161-2170. doi:10.7150/thno.23335

28. Chen S, Liu Y, Zhu S, et al. Dual-mode imaging and therapeutic effects of drug-loaded phase-transition nanoparticles combined with near-infrared laser and low-intensity ultrasound on ovarian cancer. Drug Deliv. 2018;25(1):1683-1693. doi:10.1080/ 10717544.2018.1507062

29. Capozza M, Blasi F, Valbusa G, et al. Photoacoustic imaging of integrin-overexpressing tumors using a novel ICG based contrast agent in mice. Photoacoustics. 2018;11:36-45. doi:10.1016/j. pacs.2018.07.007

30. Maturi M, Locatelli E, Monaco I, et al. Current concepts in nanostructured contrast media development for in vivo photoacoustic imaging. Biomater Sci. 2019;7(5):1746-1775. doi:10.1039/ C8BM01444B

31. Miyata A, Ishizawa T, Kamiya M, et al. Photoacoustic tomography of human hepatic malignancies using intraoperative indocyanine green fluorescence imaging. PLoS One. 2014;9(11):e112667. doi:10.1371/ journal.pone.0112667

32. Kraft JC, Ho RJY. Interactions of indocyanine green and lipid in enhancing near-infrared fluorescence properties: the basis for near-infrared imaging in vivo. Biochemistry. 2014;53(8):1275-1283. doi:10.1021/bi500021j

33. Hannah A, Luke G, Wilson K, et al. Indocyanine green-loaded photoacoustic nanodroplets: dual contrast nanoconstructs for enhanced photoacoustic and ultrasound imaging. ACS Nano. 2014;8 (1):250-259. doi: $10.1021 / \mathrm{nn} 403527 \mathrm{r}$

34. Akhtar NH, Pail O, Saran A, et al. Prostate-specific membrane antigen-based therapeutics. Adv Urol. 2012;2012:973820. doi: $10.1155 / 2012 / 973820$

35. Yu Z, Wang Y, Xu D, et al. G250 antigen-targeting drug-loaded nanobubbles combined with ultrasound targeted nanobubble destruction: a potential novel treatment for renal cell carcinoma. Int J Nanomedicine. 2020;15:81-95. doi:10.2147/IJN.S230879

36. Li M, Luo H, Zhang W, et al. Phase-shift, targeted nanoparticles for ultrasound molecular imaging by low intensity focused ultrasound irradiation. Int J Nanomedicine. 2018;13:3907-3920. doi:10.2147/ IJN.S166200

37. Haedicke K, Brand C, Omar M, et al. Sonophore labeled RGD: a targeted contrast agent for optoacoustic imaging. Photoacoustics. 2017;6(1):1-8. doi:10.1016/j.pacs.2017.03.001

38. Weber J, Beard PC, Bohndiek SE. Contrast agents for molecular photoacoustic imaging. Nat Methods. 2016;13(8):639-650. doi:10.1038/nmeth.3929 
39. Robu S, Schottelius M, Eiber M, et al. Preclinical evaluation and first patient application of $99 \mathrm{~m}$ Tc-PSMA-I\&S for SPECT imaging and radioguided surgery in prostate cancer. $\mathrm{J} \mathrm{Nucl} \mathrm{Med.} \mathrm{2017;58}$ (2):235-242. doi:10.2967/jnumed.116.178939

40. Banerjee SR, Foss CA, Horhota A, et al. 111 in- and IRDye800CWlabeled PLA-PEG nanoparticle for imaging prostate-specific membrane antigen-expressing tissues. Biomacromolecules. 2017;18 (1):201-209. doi:10.1021/acs.biomac.6b01485

41. Rauscher I, Horn T, Eiber M, et al. Novel technology of molecular radio-guidance for lymph node dissection in recurrent prostate cancer by PSMA-ligands. World J Urol. 2018;36(4):603-608. doi:10.1007/ s00345-018-2200-3

42. Lütje S, Heskamp S, Franssen GM, et al. Development and characterization of a theranostic multimodal anti-PSMA targeting agent for imaging, surgical guidance, and targeted photodynamic therapy of PSMA-expressing tumors. Theranostics. 2019;9(10):2924-2938. doi:10.7150/thno.35274
43. Kothapalli SR, Sonn GA, Choe JW, et al. Simultaneous transrectal ultrasound and photoacoustic human prostate imaging. Sci Transl Med. 2019;11(507):507. doi:10.1126/scitranslmed.aav2169

44. Garcia-Uribe A, Erpelding TN, Krumholz A, et al. Dual-modality photoacoustic and ultrasound imaging system for noninvasive sentinel lymph node detection in patients with breast cancer. Sci Rep. 2015;5(1):15748. doi:10.1038/srep15748

45. Zhu L, Guo Y, Wang L, et al. Construction of ultrasonic nanobubbles carrying CAIX polypeptides to target carcinoma cells derived from various organs. J Nanobiotechnology. 2017;15(1):63. doi:10.1186/ s12951-017-0307-0

46. Tian Y, Liu Z, Zhang L, et al. Apatinib-loaded lipid nanobubbles combined with ultrasound-targeted nanobubble destruction for synergistic treatment of HepG2 cells in vitro. Onco Targets Ther. 2018;11:4785. doi:10.2147/OTT.S170786
International Journal of Nanomedicine

\section{Publish your work in this journal}

The International Journal of Nanomedicine is an international, peerreviewed journal focusing on the application of nanotechnology in diagnostics, therapeutics, and drug delivery systems throughout the biomedical field. This journal is indexed on PubMed Central, MedLine, CAS, SciSearch ${ }^{\mathbb{R}}$, Current Contents ${ }^{\mathbb{B}} /$ Clinical Medicine, $^{2}$
Journal Citation Reports/Science Edition, EMBase, Scopus and the Elsevier Bibliographic databases. The manuscript management system is completely online and includes a very quick and fair peer-review system, which is all easy to use. Visit http://www.dovepress.com/ testimonials.php to read real quotes from published authors. 